\title{
REVIEW
}

\section{Energy transfer in plasmonic photocatalytic composites}

\author{
Xiang-Chao Ma, Ying Dai, Lin Yu and Bai-Biao Huang
}

Among the many novel photocatalytic systems developed in very recent years, plasmonic photocatalytic composites possess great potential for use in applications and are one of the most intensively investigated photocatalytic systems owing to their high solar energy utilization efficiency. In these composites, the plasmonic nanoparticles (PNPs) efficiently absorb solar light through localized surface plasmon resonance and convert it into energetic electrons and holes in the nearby semiconductor. This energy transfer from PNPs to semiconductors plays a decisive role in the overall photocatalytic performance. Thus, the underlying physical mechanism is of great scientific and technological importance and is one of the hottest topics in the area of plasmonic photocatalysts. In this review, we examine the very recent advances in understanding the energy transfer process in plasmonic photocatalytic composites, describing both the theoretical basis of this process and experimental demonstrations. The factors that affect the energy transfer efficiencies and how to improve the efficiencies to yield better photocatalytic performance are also discussed. Furthermore, comparisons are made between the various energy transfer processes, emphasizing their limitations/benefits for efficient operation of plasmonic photocatalysts.

Light: Science \& Applications (2016) 5, e16017; doi:10.1038/Isa.2016.17; published online 12 February 2016

Keywords: hot electron injection; near-field enhancement; plasmonic photocatalyst; resonance energy transfer; surface plasmon resonance

\section{INTRODUCTION}

Since the report on water splitting over titanium dioxide $\left(\mathrm{TiO}_{2}\right)$ by Fujishima et al. ${ }^{1}$, semiconductor photocatalysts have received considerable attention in the field of solar energy conversion and utilization ${ }^{2-4}$. In conventional semiconductor photocatalysts, the semiconductor absorbs light by inter-band excitation of electrons, which generates electron-hole pairs. Then, the generated electrons and holes may be transferred to the surface of the semiconductor, where they initiate redox reactions. During the transfer process, the electrons and holes may also recombine with each other, which have no effect on the photocatalysis. Over the past 40 years, the main limitations of using semiconductor photocatalysts, such as $\mathrm{TiO}_{2}$ and iron(III) oxide $\left(\mathrm{Fe}_{2} \mathrm{O}_{3}\right)$, for large-scale applications have been the short-range light response, poor light absorption rate, and high recombination rate of photogenerated carriers, which result in low solar energy conversion efficiencies. Despite the considerable efforts to overcome these deficiencies, including extrinsic and intrinsic doping ${ }^{5-10}$, sensitization $^{11-13}$, and surface treatment ${ }^{14-17}$, significant breakthroughs have not yet been achieved.

In the recent efforts to develop photocatalytic materials, researchers tended to develop novel photocatalytic systems, such as the plasmonic photocatalyst $^{18,19}$, ferroelectric photocatalyst ${ }^{20-23}$, and graphenebased photocatalyst ${ }^{24,25}$, based on some basic physical principles. The plasmonic photocatalyst generally exhibits wide range and very efficient light absorption. Moreover, the exotic approach for generating electron-hole pairs often leads to a low carrier recombination rate. Thus, it has the greatest potential for improving many of the intrinsic limitations of conventional semiconductor photocatalysts ${ }^{13,26,27}$. Following the development of the 'plasmonic photocatalyst' (Ag/ $\mathrm{SiO}_{2} / \mathrm{TiO}_{2}{ }^{18}$ and $\mathrm{Ag} / \mathrm{AgCl}^{19}$ ) in 2008, it has received substantial interest, and many plasmonic photocatalysts have been developed ${ }^{28-36}$. For example, by depositing noble metal plasmonic nanoparticles (PNPs) onto conventional semiconductor photocatalysts (e.g., $\mathrm{TiO}_{2}$, cadmium sulfide (CdS), and $\mathrm{Fe}_{2} \mathrm{O}_{3}$ ) in different ways ${ }^{37-53}$, researchers have developed various plasmonic photocatalytic composites that exhibit significantly enhanced photocatalytic activities. In these composites, the PNPs efficiently absorb visible light through localized surface plasmon resonance (LSPR) and convert it into holes and electrons in the nearby semiconductors. Thanks to the intensive research over the past century, the physics about the LSPR of PNPs is well understood $^{54,55}$. However, its role in plasmonic photocatalysts and particularly in the energy transfer from PNPs to semiconductors was mysterious when the system was developed. The LSPR-related energy transfer is an essential step in the plasmonic photocatalytic process, and it plays a vital role in determining the final photocatalytic performance. Therefore, the details of the energy transfer are of great scientific importance for understanding the excellent photocatalytic performance; technologically, they can also provide fundamental guidelines for optimizing the existing plasmonic systems and for developing new ones. Over the past several years, considerable experimental and theoretical efforts have been devoted to understanding this question and rapid progress is still being made.

There are many reviews in the literature on the applications and experimental preparation of plasmonic photocatalysts ${ }^{27-34,56}$. However, to the 
best of our knowledge, a systematic review on the energy transfer mechanism in plasmonic photocatalytic composites has not yet been published. This work is thus aimed at filling this gap. It begins with a brief introduction to the fundamental properties of the LSPR of PNPs, which is necessary to understand the energy transfer mechanism. Then, we present detailed discussions about the three energy transfer processes proposed thus far. These processes are the plasmon-induced hot electron injection, plasmon-induced resonance energy transfer, and plasmon-induced radiative energy transfer. In addition, in this review, we tentatively propose a new energy transfer process, called plasmon-induced direct electron injection. Finally, we end with a conclusion and an outlook on investigating the energy transfer processes for optimizing the plasmonic photocatalytic activity.

\section{FUNDAMENTAL PROPERTIES OF LSPR}

The LSPR of PNPs can include many plasmon modes, such as the dipole plasmon mode, quadrupole mode and considerably higher plasmon modes ${ }^{55,57}$. For photocatalytic applications, the most relevant mode is the dipole plasmon resonance. Therefore, in the present review, LSPR primarily refers to the dipole plasmon mode, which describes the collective oscillation of the conduction electrons in PNPs in response to the incident light. Upon light irradiation, the electric field will displace the conduction electrons relative to the nuclei, inducing a large electric dipole. Simultaneously, a restoring force arises because of the Coulomb attraction between electrons and nuclei, which results in resonant oscillation of the conduction electrons at a certain frequency. The LSPR generally occurs when the PNPs are considerably smaller than the wavelength of the incident light, as schematically illustrated in Figure 1.

The LSPR of PNPs can lead to very efficient absorption of light. For example, the absorption cross section of LSPR can be several or even tens of times greater than the physical cross section. As shown in Figure 1, LSPR can induce a large electric dipole, which oscillates at the same frequency as that of the electric field of the incident light ${ }^{30,55}$. This electric dipole can result in an intense electric field near the PNP, whose intensity can be tens to thousands of times greater than that of incident light $\mathrm{t}^{26,55,58}$. This is the so-called near-field enhancement phenomenon. Because of the physical origin of near-field enhancement, it decays quickly when moving away from the PNP and is thus limited to the very vicinity of the PNP. As widely demonstrated, both the LSPR wavelength and absorption efficiency can be easily tuned by changing the sizes, shapes, compositions, and dielectric environments of $\mathrm{PNPs}^{26,58-60}$. Moreover, both the intensity and distribution range of

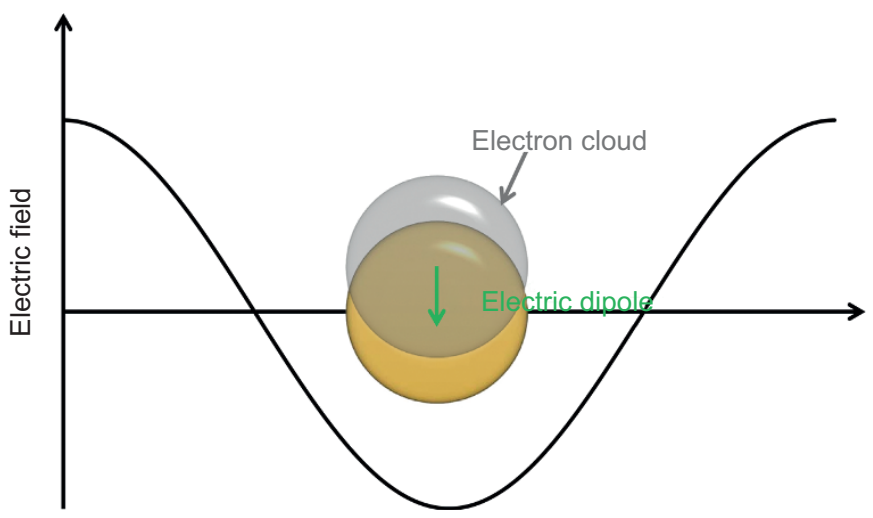

Figure 1 Schematic illustration of LSPR for a plasmonic nanosphere. LSPR, localized surface plasmon resonance. the near-field change accordingly. These properties of LSPR can lead to various energy transfer mechanisms from PNPs to semiconductors and provide versatile means for tuning the efficiency of each energy transfer process, which will be discussed in the following.

\section{PLASMON-INDUCED HOT ELECTRON INJECTION FROM PLASMONIC METAL TO SEMICONDUCTOR}

The generation of hot electrons in PNPs has been studied for several decades ${ }^{27,34}$. Here, the term 'hot electron' refers to electrons that are not in thermodynamic equilibrium with the atoms in a material. As introduced in the previous section, LSPR is characterized as the collective coherent oscillation of the conduction electrons in PNPs. Moreover, there exist two competitive ways for the dephasing of the resonant oscillation, namely, radiative emission of photons and nonradiative relaxation through electron-electron, electron-phonon, electron-surface, or electron-adsorbate scattering (Figure 2a, left ${ }^{59,61-64}$. The hot electrons are generated during the non-radiative relaxation process primarily through electron-electron scattering, which results in intra- and inter-band excitation of the conduction electrons (Figure 2a, right). Note that the intra- and inter-band transition requires an energy overlap between the LSPR frequency and the transition energy. For $\mathrm{Au}$, the threshold for the $d$ to $s$ inter-band transition is approximately $2.4 \mathrm{eV}$, which corresponds to a wavelength smaller than its LSPR wavelength at approximately $550 \mathrm{~nm}$ or longer

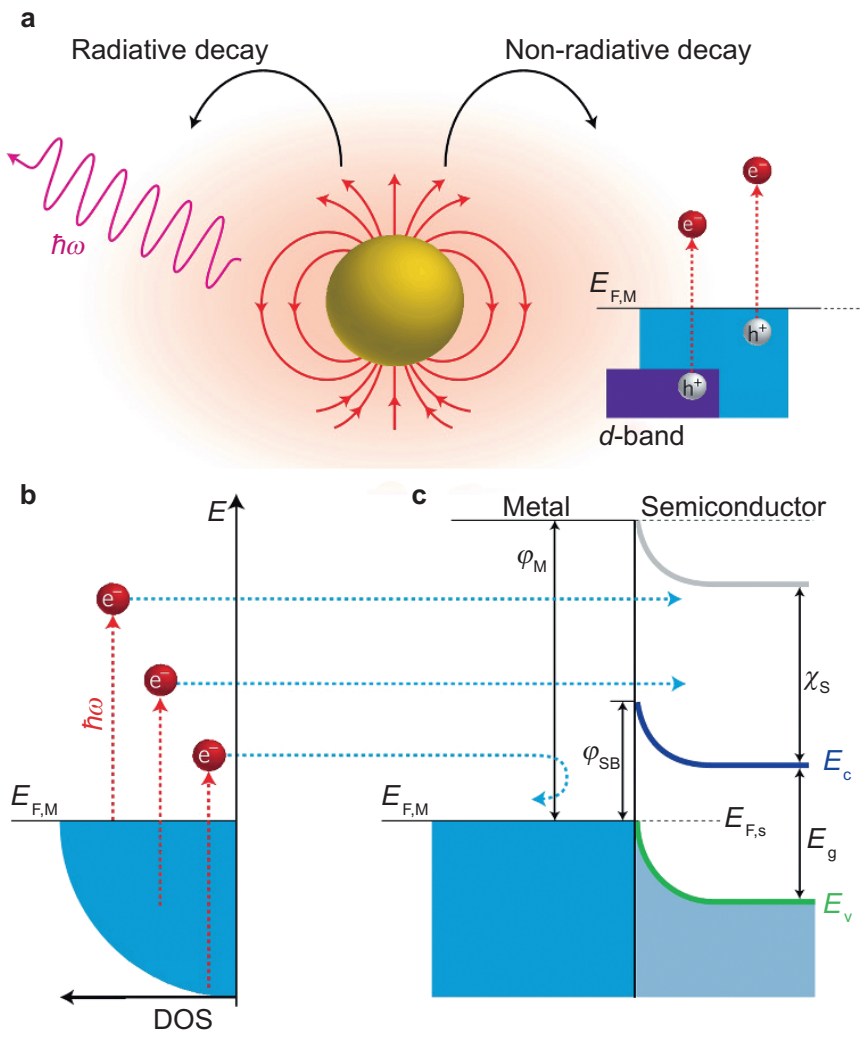

Figure 2 (a) LSPR can decay radiatively or non-radiatively. The radiative decay emits photons. During the non-radiative decay, hot electrons may be generated via intra- and inter-band excitation of the conduction electrons. (b) The energies of hot electrons have a wide distribution range. (c) The hot electrons with large energy can surmount the interface Schottky barrier, thus being injected into the conduction band of the semiconductor. Figure reproduced with permission: Ref. 27, (C) 2014 NPG. LSPR, localized surface plasmon resonance; DOS, density of states. 
wavelengths owing to the large dielectric constants of semiconductor substrates or special shapes of PNPs ${ }^{26,53,65}$. Consequently, the generation of hot electrons occurs primarily through the intra-band transitions in Au PNPs. In addition, the LSPR overlaps with intra-band excitations in $\mathrm{Ag}$, which is also beneficial for generating reasonably high energy hot electrons. The energies of hot electrons have a wide distribution range (Figure $2 \mathrm{~b}$ ). It has been reported that the energies of hot electrons are approximately between $1 \mathrm{eV}$ and $4 \mathrm{eV}$ for $\mathrm{Au}$ and $\mathrm{Ag}$ $\mathrm{PNPs}^{26,66}$. Such energetic hot electrons may migrate to the adjacent semiconductor if their energies are larger than the Schottky barrier $(\mathrm{SB})$ at the interface between the metal and semiconductor (Figure 2c). Moreover, although the energies of some hot electrons are not large enough to surmount the SB, they may also be injected into the semiconductor through the tunneling effect, as proposed by Mubeen et al. ${ }^{67}$ As we can see, this hot electron injection mechanism is very similar to that of a dye-sensitized solar cell ${ }^{13,68,69}$.

Recently, the Atwater and Louie groups theoretically investigated the generation of hot carriers from the decay of surface plasmon polaritons (SPPs) on metal-dielectric interfaces ${ }^{70,71}$. They noted that $\mathrm{Au}$ is beneficial for generating very hot holes rather than energetic hot electrons because of the special electronic structures. However, note that in their calculations, the energies of some modes of SPPs were large enough to excite the $d$ to $s$ inter-band transitions. This is often not true in the case of LSPR, whose energies are generally too small to initiate the inter-band transitions. Furthermore, the

a
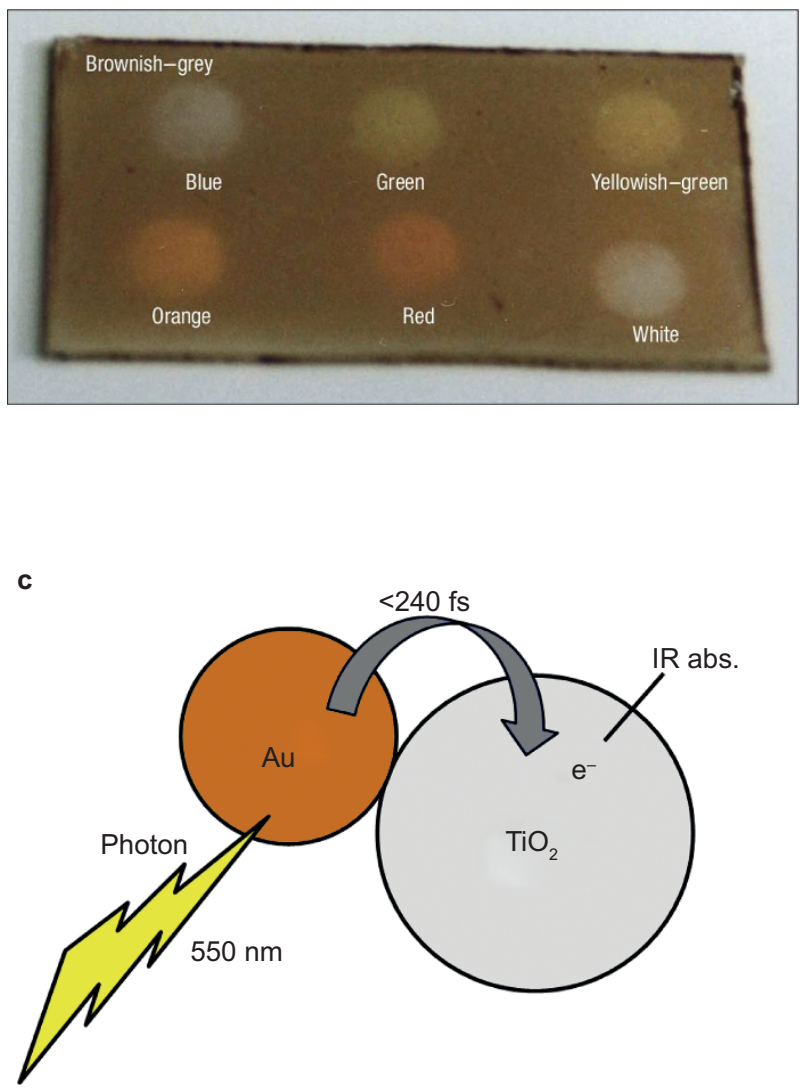

geometry effect of confined PNPs on promoting intra-band transitions and thus forming hot electrons was also demonstrated by the Atwater group ${ }^{70}$.

Experimentally, Zhao et al. first reported hints about plasmoninduced hot electron injection from $\mathrm{Au} / \mathrm{Ag}$ to $\mathrm{TiO}_{2}{ }^{72}$. When the incident light overlapped with the LSPR absorption of Au/Ag PNPs, they observed an increase in the anodic photocurrent for a $\mathrm{TiO}_{2}$ film electrode containing dispersed $\mathrm{Au} / \mathrm{Ag}$ PNPs. A breakthrough was achieved in 2003, when Ohko et al. reported the multicolor photochromism of a $\mathrm{TiO}_{2}$ film containing Ag PNPs (Figure 3a), which provides strong evidence for the electron exchange between $\mathrm{Ag}$ and $\mathrm{TiO}_{2}$ upon light irradiation $^{73}$. Direct observation of the injection of hot electrons into the conduction band of $\mathrm{TiO}_{2}$ was realized by Furube et al. ${ }^{74}$ Because strong intra-band absorption in the infrared (IR) range can be observed when electrons are injected into the conduction band of $\mathrm{TiO}_{2}{ }^{75}$, they used femtosecond-resolved IR probing to detect any possible electron transfer from $\mathrm{Au}$ to $\mathrm{TiO}_{2}$. Indeed, IR absorption was observed, and the ultrafast hot electron transfer from Au nanodots to $\mathrm{TiO}_{2}$ was determined to be within 240 fs (Figure 3b and 3c). Subsequently, many experiments have also demonstrated the injection of hot electrons from PNPs to semiconductors, and different methods have been developed to probe the electron transfer ${ }^{37,50,76-79}$. For example, in a very recent study, using $\mathrm{Ag}^{+}$from $\mathrm{AgNO}_{3}$ as electron probe ions and sodium citrate as a positive charge scavenger, Li et al. showed that $\mathrm{Ag}$ nanoparticles only form on the $\mathrm{TiO}_{2}$ shell when the

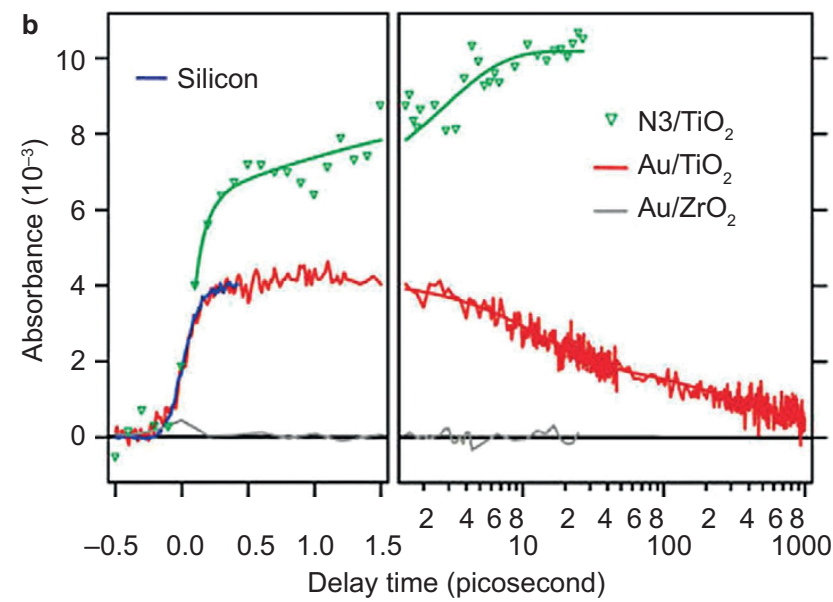

d

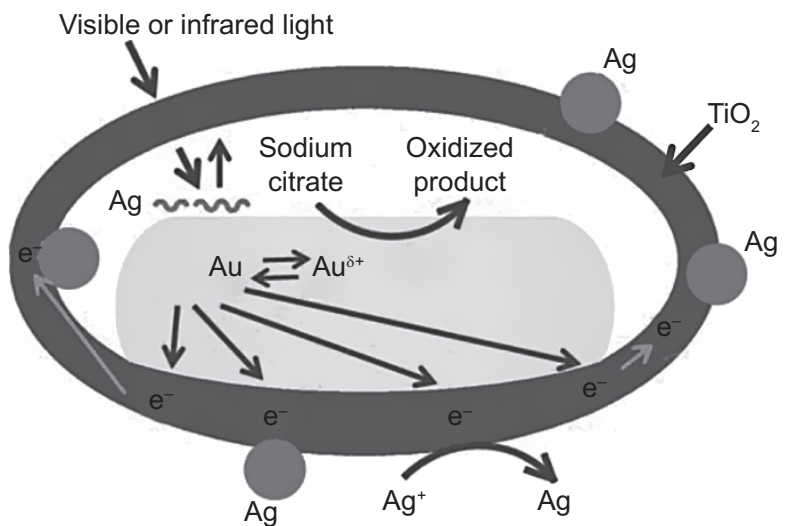

Figure 3 Experimental verifications of the hot electron injection energy transfer. (a) Photochromism of $\mathrm{TiO}_{2}$ thin film dispersed with Ag PNPs, which originates from the electron exchange between $\mathrm{Ag}$ and $\mathrm{TiO}_{2}$. (b) IR absorption of $\mathrm{Au} / \mathrm{TiO}_{2}$ in comparison with $\mathrm{N} 3 / \mathrm{TiO}_{2}$ and $\mathrm{Au} / \mathrm{ZrO} \mathrm{Z}_{2}$. (c) Experimentally determined electron injection process and timescale. (d) Probe reaction of $\mathrm{Ag}^{+}$, which is used to detect electrons. Figure reproduced with permission: a, Ref. 73, ๑ 2003 NPG; b, c, Ref. 74, ๑ 2007 ACS; d, Ref. 79, (c) 2015 Wiley-VCH. IR, infrared. 
$\mathrm{Au} / \mathrm{TiO}_{2}$ is irradiated by light with the LSPR wavelength of $\mathrm{Au}$, which confirms the injection of hot electrons from Au to $\mathrm{TiO}_{2}$ (Figure 3d) ${ }^{79}$.

As shown in Figure 2c, when a metal is brought into contact with an $n$-type semiconductor, an interface SB is formed. For a high rate of hot electron injection, the energies of the hot electrons must surpass the barrier. Considerable efforts are thus expended to theoretically study this dynamic process. For $\mathrm{Au} / \mathrm{TiO}_{2}$, a moderate $\mathrm{SB}$ of approximately 1 $\mathrm{eV}$ is formed according to the Schottky-Mott theory ${ }^{30,80}$. Because the energies of hot electrons typically range between $1 \mathrm{eV}$ and $4 \mathrm{eV}$ above the Fermi level, the small SB makes efficient electron transfer from $\mathrm{Au}$ to $\mathrm{TiO}_{2}$ energetically possible, which also renders $\mathrm{Au} / \mathrm{TiO}_{2}$ a model system for studying and utilizing the hot electron injection process. Due to the suitable band alignments, hot electron injection has also been observed in many other PNP-sensitized semiconductors, such as $\mathrm{Ag} / \mathrm{TiO}_{2}, \mathrm{Au} / \mathrm{ZnO}, \mathrm{Au} / \mathrm{WO}_{3}$, and $\mathrm{Au} / \mathrm{CdS}$ (Figure 4a) ( $^{37,50,78,81}$. However, a small SB does not necessarily ensure hot electron transfer. For example, hot electron transfer has never been experimentally observed in $\mathrm{Au} / \mathrm{Fe}_{2} \mathrm{O}_{3}$, although the $\mathrm{SB}$ of $\mathrm{Au} / \mathrm{Fe}_{2} \mathrm{O}_{3}$ is considerably smaller than that of $\mathrm{Au} / \mathrm{TiO}_{2}{ }^{82}$. This result indicates that some unknown factors may also determine the hot electron transfer in addition to the SB. In a recent theoretical study, Long et al. showed that effective hybridization of the unoccupied states in the metal and semiconductor is very important for efficient injection of hot electrons from the metal to semiconductor (Figure $4 \mathrm{~b}$ and $4 \mathrm{c})^{52}$.

The solar utilization efficiency through the hot electron injection is generally very low. For example, Mubeen et al. elaborately devised an autonomous plasmonic solar water splitter that consisted of gold nanorods and a crystalline $\mathrm{TiO}_{2}$ layer, in which all of the electrons and holes originate from the hot electron injection energy transfer (Figure $5 a)^{51}$. They showed that the solar to energy conversion efficiency is only approximately $0.1 \%$. For practical applications, this conversion efficiency should be enhanced much further. Based on the mechanism of hot electron injection, there are two important factors that can affect the solar utilization efficiency, namely, the hot electron generation efficiency (HEGE) and the hot electron injection efficiency (HEIE). Here, HEGE refers to the efficiency of converting LSPR into energetic hot electrons moving to the semiconductor. In some samples, the PNPs are relatively large (e.g., larger than $40 \mathrm{~nm}$ ), which leads to a low HEGE primarily due to the prominent scattering effect. Although the generation of hot electrons from the decay of LSPR is very efficient in small PNPs, the portion of hot electrons moving toward the semiconductor is still low provided that the momentum-direction distribution of hot electrons is approximately isotropic and that the PNPs are generally atop the semiconductor.

Over the past few years, researchers have found a number of ways to improve the HEGE. Giugni et al. showed that the HEGE can be significantly increased through adiabatic compression of SPPs in a nanocone of $\mathrm{Au}^{83}$. This is because the special structure can efficiently transform the propagating wave of SPPs into a quasi-static local field and then into hot electrons moving to the semiconductor (Figure 5b). In fact, combining SPPs with a semiconductor photocatalyst can provide other means for enhancing the photocatalysis. For example, by elaborately designing a hybrid structure of $\mathrm{Au} / \mathrm{Fe}_{2} \mathrm{O}_{3}$, Li et al. showed that the SPPs can significantly enhance the photocatalysis through confining the light in $\mathrm{Fe}_{2} \mathrm{O}_{3}$ and thus increasing its light absorption efficiency ${ }^{84}$. In a photodetection device composed of $\mathrm{Au} / \mathrm{Si}$, Knight et al. showed that a photocurrent maximum is generated at the LSPR peak wavelength (Figure $5 c)^{85}$. This suggests that the HEGE is enhanced when the LSPR absorption efficiency increases. The surrounding medium also plays an important role in the HEGE. It was shown that the $1 \mathrm{~nm}$ Ti metal interface between $\mathrm{Au}$ and $\mathrm{Si}$ is responsible for producing $33 \%$ of the hot electrons, which can increase to more than $50 \%$ for a thickness of $5 \mathrm{~nm}^{85}$.

For a specific plasmonic system, the composite structure also affects the HEGE. As shown in Figure 6a, upper, when the PNP is atop a semiconductor, only the hot electrons with momentum falling within the small red shaded cone in the momentum space may be injected into the semiconductor. This can be significantly improved if one embeds the PNP into the semiconductor to some extent, as show in Figure $6 \mathrm{a}$, lower. Then, the hot electrons with momentum falling within the large red-shaded cone may be injected into the semiconductor. Therefore, considerably higher photocatalytic activities have been reported for the partially or fully embedded plasmonic photocatalytic systems ${ }^{43,67,79,86-88}$. It has recently been proposed that the Janus structure may promote the generation of hot electrons with momentum mainly pointing toward the semiconductor, thus also
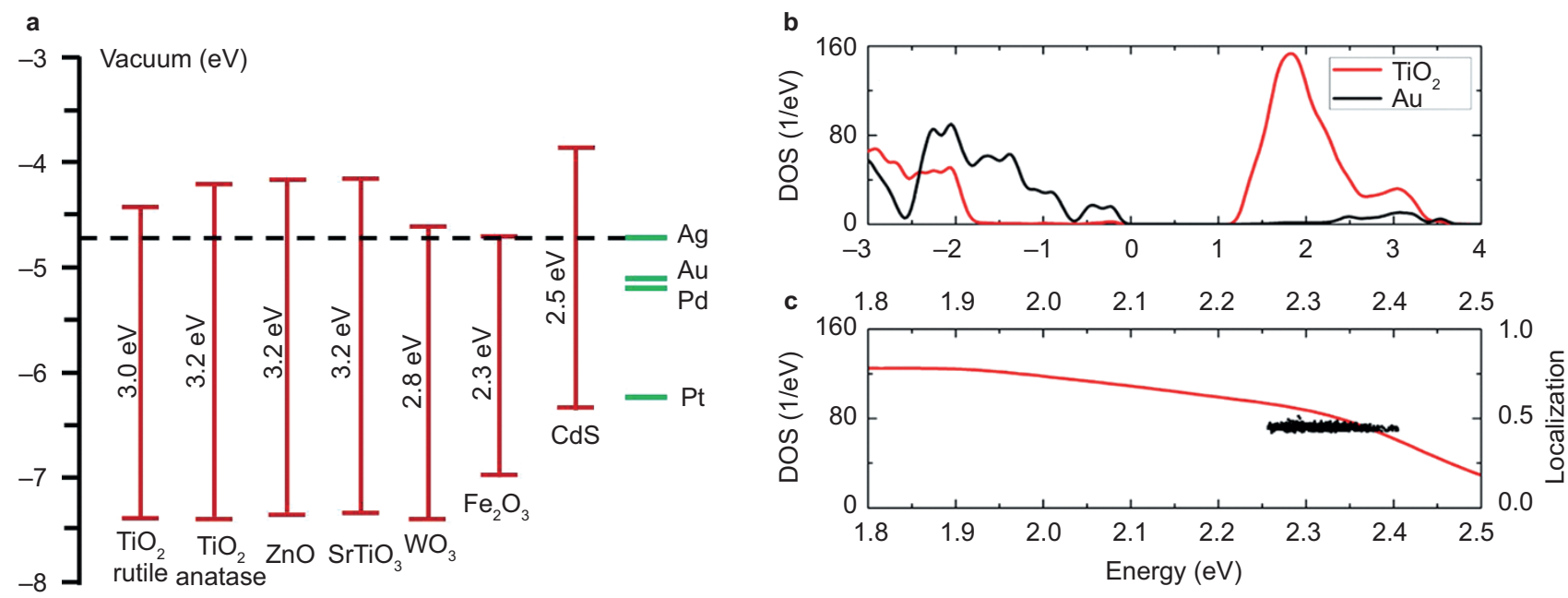

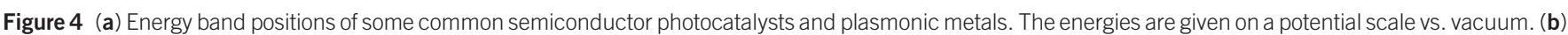

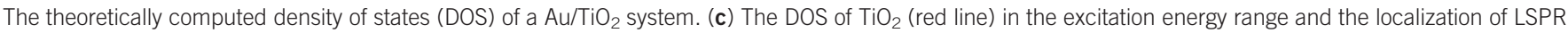

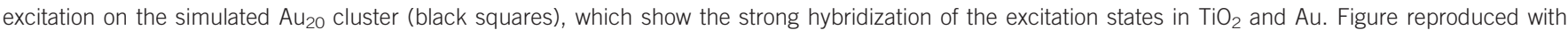
permission: b, c, Ref. 52, (c) 2014 ACS. 
a

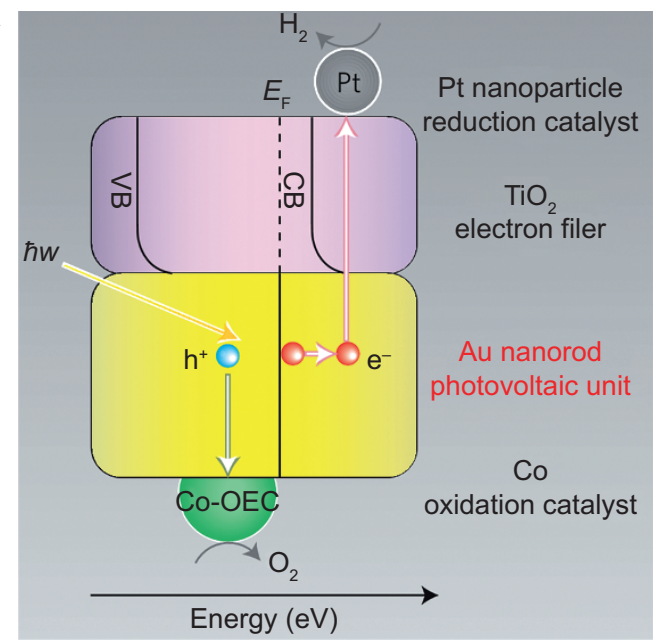

b

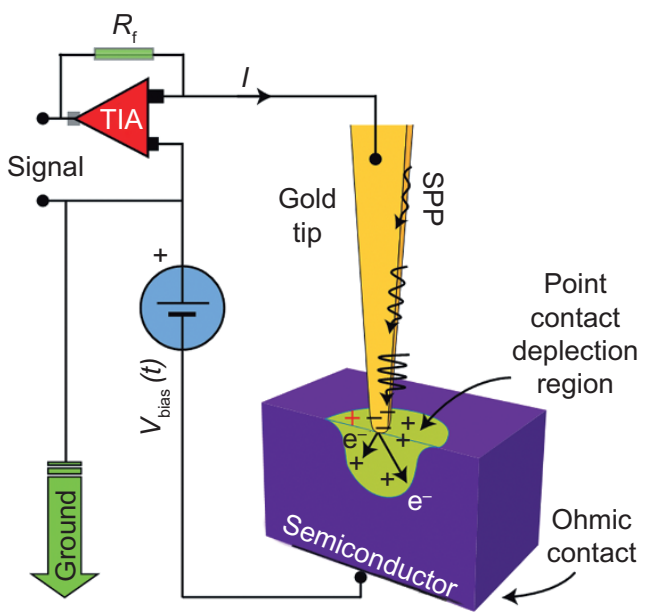

c Photocurrent spectra (a.u)

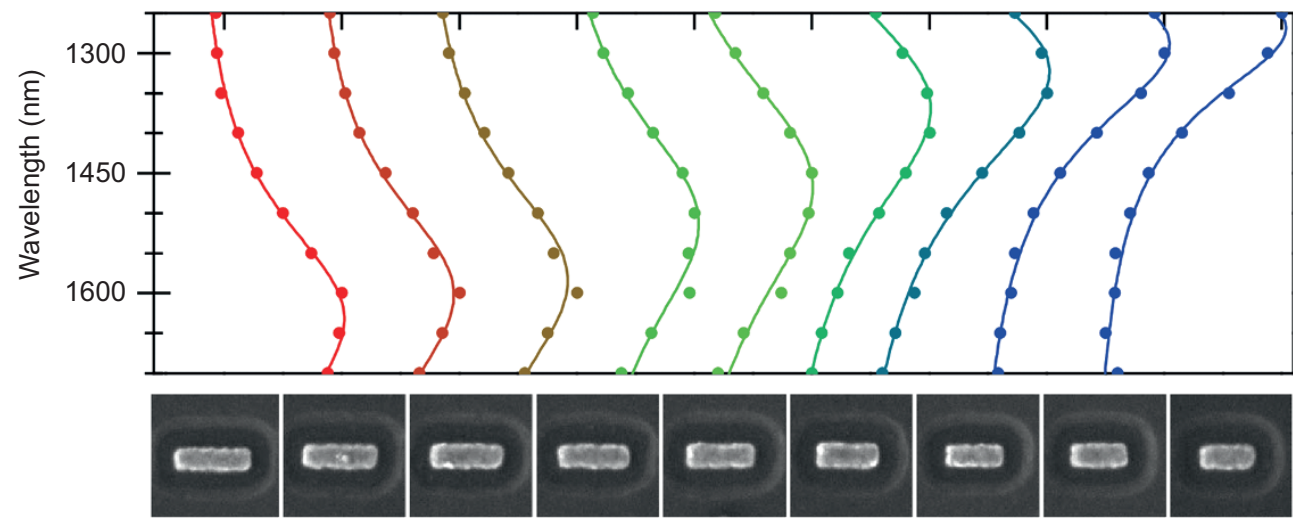

Absorption (a.u.)

Figure 5 (a) Energy level diagram showing the generation and transfer of carriers in the plasmonic solar water splitter devised by Mubeen et al. (b) Schematic of the setup devised by Giugni et al. (c) Photocurrent spectra for a specific Au antenna, which is consistent with the calculated absorption spectra due to LSPR. Figure reproduced with permission: a, Ref. 51, (c) 2013 NPG; b, Ref. 83, (c) 2013 NPG; c, Ref. 85, (c) 2011 AAAS. LSPR, localized surface plasmon resonance; SPP, surface plasmon polariton.

increasing the $\mathrm{HEGE}^{48,89,90,91}$. In addition, for a free-electron metal, the momentum-direction of generated hot electrons should mainly be along the oscillation direction of LSPR according to momentum conservation. Therefore, any structures that facilitate oscillation of LSPR along the interface normal to the metal-semiconductor composite will primarily generate hot electrons that move to the semiconductor and thus increase the HEGE.

Theoretically, the HEIE is generally described by a modified Fowler equation $^{83,86}$, which indicates that the HEIE decreases with increasing $\mathrm{SB}$ or with decreasing hot electron energy relative to the SB. For example, because the conduction bands of $\mathrm{ZrO}_{2} / \mathrm{SiO}_{2}$ lie much higher in energy than that of $\mathrm{TiO}_{2}$, which results in a larger interface SB in $\mathrm{Au} /$ $\mathrm{ZrO}_{2}$ and $\mathrm{Au} / \mathrm{SiO}_{2}$, there is essentially no hot electron injection in these systems ${ }^{74,85}$. Li et al. also recently demonstrated the effect of hot electron energy relative to the SB on the HEIE by comparing the hot electron transfer properties in $\mathrm{Au}-\mathrm{TiO}_{2}$ and $\mathrm{CdS}-\mathrm{Au}-\mathrm{TiO}_{2}{ }^{92}$. For a specific metal-semiconductor system, the interface SB depends strongly on the interfacial chemical and electronic structures ${ }^{91,93-95}$. This provides plenty of room to optimize the interface SB for improving HEIE. For instance, Tamura et al. recently demonstrated that the interface $\mathrm{SB}$ of $\mathrm{Pt} / \mathrm{TiO}_{2}$ is strongly affected by the interface oxygen deficiency ${ }^{95}$. They showed that the interface contact type can be changed from a Schottky- to Ohmic-type contact by varying the interface oxygen deficiency (Figure 6b). Our group also demonstrated that the replacement of $\mathrm{O}$ by $\mathrm{Au}$ atoms at the $\mathrm{Au} / \mathrm{TiO}_{2}$ interface can increase the interface SB (Figure $6 \mathrm{c})^{91}$. In addition, because hot electrons lose energy very quickly through electron-electron or electron-phonon scattering, it is better to achieve ultrafast hot electron extraction for improving the HEIE. This factor has been highlighted in recent reviews ${ }^{27,34,96}$. Thus, we do not discuss it in detail.

Although the hot electron injection energy transfer process can generate electron-hole pairs using visible or even IR light, which increases the solar energy utilization, there are many limitations on this energy transfer process for photocatalytic applications. As shown above, this energy transfer process requires proper band alignments and efficient electronic coupling between PNPs and the semiconductor to ensure an efficient HEIE. Thus, direct contact between PNPs and the semiconductor is required. Consequently, some plasmonic metals such as $\mathrm{Ag}$ and $\mathrm{Cu}$, which are easily degraded, are not applicable for supporting this energy transfer process. Moreover, upon the injection of hot electrons, a large amount of hot holes are left in the PNPs. These holes must be immediately compensated to retain a 
a

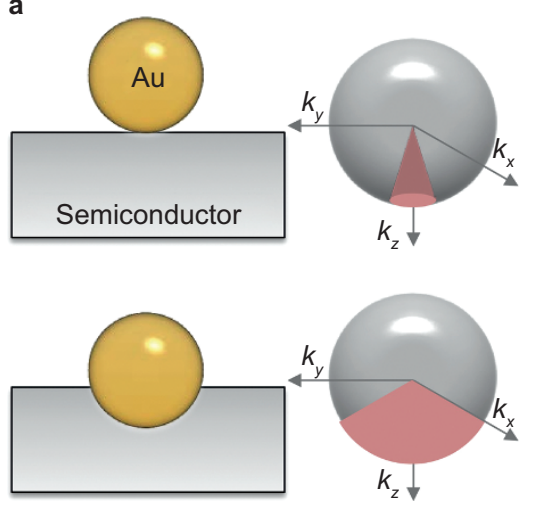

b

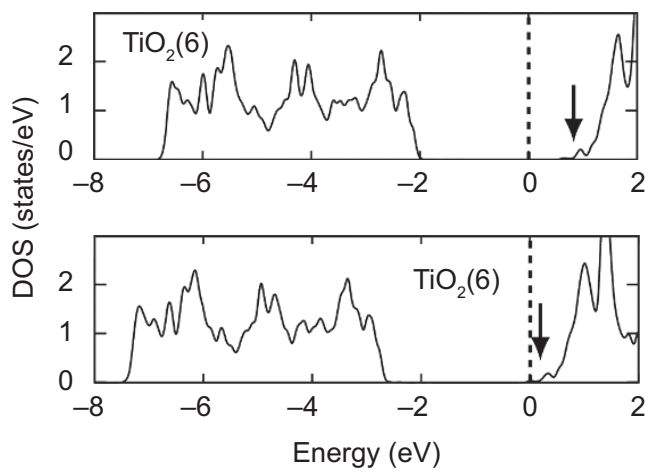

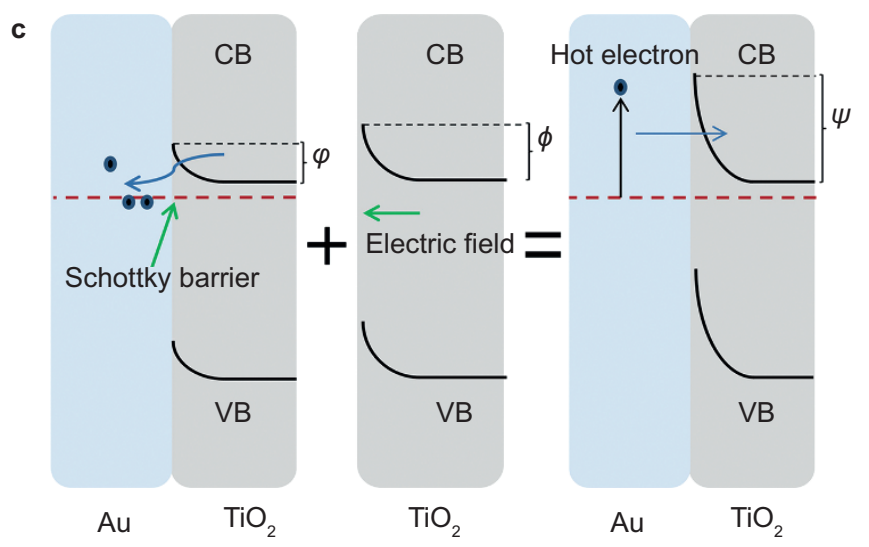

Figure 6 (a) The hot electrons can transport only through the small bottom interface when the PNP is atop the semiconductor (upper); the interface area through which the hot electrons can transfer into the semiconductor is significantly increased when the PNP is partially embedded into the semiconductor (lower). (b) The Fermi levels relative to the conduction band of $\mathrm{TiO}_{2}$ for stoichiometric (upper) and fully reduced (lower) $\mathrm{Pt}_{\mathrm{TiO}} \mathrm{Tinterfaces}_{2}$. (c) The band bending induced by replacing O with Au atoms at the interface, which further increases the interface SB. Figure reproduced with permission: b, Ref. 95, ( 2009 APS; c, Ref. 91 , ( 2014 ACS.

sustainable LSPR. Therefore, an electron donor or hole-transporting material should be in contact with the PNPs. This is the so-called charge balance requirement ${ }^{27}$. These requirements of band alignment, electronic coupling and charge balance can impose strict limitations on the selection of materials for preparing efficient plasmonic photocatalysts. In addition, to prevent the injected hot electrons from flowing readily back into the PNPs, upward band bending in the semiconductor is required (Figure 2c). This means that only $n$-type semiconductors are preferred. Very recently, Lin et al. demonstrated that under UV-Vis light irradiation, the injection of hot electrons from Au to $\mathrm{TiO}_{2}$ shortens the lifetime of electrons generated upon UV light irradiation in $\mathrm{TiO}_{2}{ }^{97}$. This result indicates that the hot electron injection may also have a negative effect on the intrinsic photocatalytic activity of conventional photocatalysts.

\section{PLASMON-INDUCED DIRECT ELECTRON INJECTION FROM PLASMONIC METAL TO SEMICONDUCTOR}

When the dimension of PNPs is comparable to or smaller than the electronic mean free path and there are adsorbates on the PNPs, another ultrafast dephasing channel for LSPR, known as chemical interface damping, may dominate. In this dephasing channel, the interaction of LSPR with adsorbates induces dephasing through the direct excitation of resonant electrons into the unoccupied states of adsorbates ${ }^{98,99}$. Note that in this process, energy conservation has to be satisfied, that is, the energy of the unoccupied states of adsorbates relative to the Fermi level of the plasmonic metal must overlap with the LSPR energy. This direct electron injection to adsorbates has been widely demonstrated in atoms or small molecules adsorbed PNP systems ${ }^{100-102}$, for which an excellent review by Christopher et al. was recently published ${ }^{103}$. Similarly, it is highly expected that this mechanism also exists in plasmonic photocatalytic composites. Experientially, it has been suggested to exist in $\mathrm{Ag} / \mathrm{SiO}_{2}{ }^{99}$.

Compared with the hot electron injection mechanism, this is a potentially more efficient mechanism that overcomes the momentum, energy, and time scale requirements of hot electron transfer. However, for this energy transfer mechanism to efficiently contribute to the photocatalytic activity of plasmonic metal-semiconductor composites, the interfacial unoccupied states to which the resonant electrons are excited must lie higher in energy than the conduction band of the semiconductor far from the interface to facilitate the transfer of injected electrons out of the interfacial region where they cannot initiate redox reactions (see Figure 7). At present, the interfacial electronic structures between PNPs and semiconductors are poorly understood. There are also few experimental reports in the literature about this plasmon-induced direct electron injection. Therefore, if and how this direct electron injection mechanism functions in the plasmonic composite require further studies in the future.

\section{PLASMON-INDUCED RESONANCE ENERGY TRANSFER}

Among researchers studying plasmonic photocatalysts, two different terms are generally used to refer to the plasmon-induced resonance energy transfer. Here, we discuss them separately. 


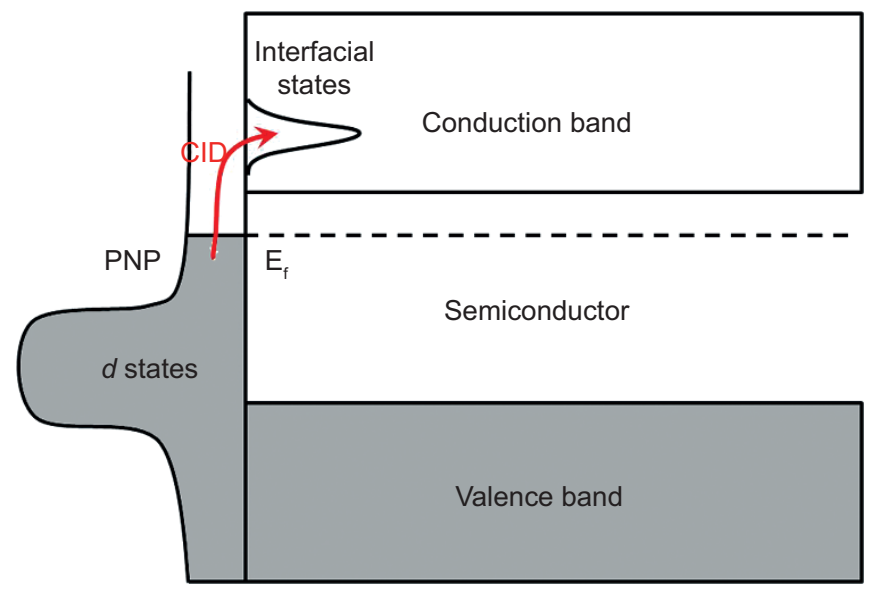

Figure 7 Schematic illustration of direct electron injection from a PNP to a semiconductor via CID. PNP, plasmonic nanoparticle; CID, chemical interface damping.

\section{ENERGY TRANSFER THROUGH NEAR-FIELD ENHANCEMENT}

The optical absorption mechanism of a semiconductor is generally described by Fermi's golden rule in the electric dipole approximation as follows ${ }^{58,104}$ :

$$
w_{k \rightarrow m}^{0}=\left(2 \pi e^{2} / \hbar\right) E_{0}^{2}\left|p_{m k}\right|^{2} \delta\left(\mathrm{E}_{m}-\mathrm{E}_{k}-\hbar \omega\right)
$$

where $E_{0}^{2}$ is the intensity of the electric field of incident light, $\left|p_{m k}\right|$ is the momentum matrix element in the semiconductor, and $\mathrm{E}_{k}$ and $\mathrm{E}_{m}$ are the energies of the initial and final states in the electron transition, respectively. As shown, the absorbance increases linearly with the intensity of the perturbation electric field. In the section 'Fundamental Properties of LSPR', we indicated that the plasmonic dipole can induce near-field enhancement. Electromagnetic simulations using the finite-difference time-domain (FDTD) or discrete dipole approximation (DDA) method revealed that the intensity of the plasmon-induced near-field can reach tens to thousands of times that of the incident light ${ }^{26,55,58}$. Thus, the near-field can significantly enhance the inter-band or other optical transitions in the nearby semiconductor if the LSPR spectra overlap with them. The full enhancement (FE) of light absorption can be estimated using the following equation:

$$
\mathrm{FE}=\frac{\int_{V} d z \int d x d y|E(x, y, z)|^{2}}{\int_{V} d z \int d x d y\left|E_{0}\right|^{2}}
$$

where the integrating volume $V$ refers to the volume in the semiconductor covered by the near-field. $E(x, y, z)$ is the value of the near-field at point $(x, y, z)$, and $E_{0}$ is the electric field of incident light. This energy transfer through near-field enhancement is easy to understand and has been widely demonstrated experimentally ${ }^{18,45,46,58,65}$, for which some exhaustive reviews are available $e^{26,32,96}$. Here, we do not discuss them in detail.

\section{ENERGY TRANSFER THROUGH DIPOLE-DIPOLE COUPLING}

Another term for the plasmonic resonance energy transfer was proposed by Cushing et al. in $2012^{104}$. From their perspective, the plasmonic resonance excites electron-hole pairs in the semiconductor by coupling of the large plasmonic dipole to the electron-hole pair dipole in the semiconductor. This picture is very similar to the F-rster resonance energy transfer, in which the fluorescent donor is replaced by the plasmonic dipole ${ }^{105}$. In their original work, core-shell $\mathrm{Au} @ \mathrm{Cu}_{2} \mathrm{O}$ and sandwiched $\mathrm{Au} @ \mathrm{SiO}_{2} @ \mathrm{Cu}_{2} \mathrm{O}$ particles were elaborately prepared to isolate the various plasmonic energy transfer processes. Because of the insertion of an insulating silica layer in $\mathrm{Au} @ \mathrm{SiO}_{2} @ \mathrm{Cu}_{2} \mathrm{O}$, the related electron injection energy transfer is ruled out. As shown in Figure 8a and 8b, the photocatalytic action spectra of $\mathrm{Au} @ \mathrm{Cu}_{2} \mathrm{O}$ and $\mathrm{Au} @ \mathrm{SiO}_{2} @ \mathrm{Cu}_{2} \mathrm{O}$ follow the trend of the extinction spectra of the corresponding systems. Thus, the involvement of LSPR in creating electron-hole pairs is verified. Moreover, the results of transient-absorption spectroscopy show that the number of carriers generated in $\mathrm{Cu}_{2} \mathrm{O}$ obeys the dipole-dipole interaction rules (Figure $8 \mathrm{c}$ and $8 \mathrm{~d}$ ). Therefore, they proposed that the energy transfer occurs through dipole-dipole coupling.

This energy transfer mechanism is based on the following physics. As introduced above, the interaction of the semiconductor with an electromagnetic field is described in the electric dipole approximation. From the semi-classical perturbation theory, the interaction Hamiltonian between a dipole $\vec{\mu}$ and electric field $\vec{E}$ can be expressed as $H^{\prime}=-\vec{\mu} \bullet \vec{E}^{106}$. Replacing $\vec{E}$ with the electric field induced by the plasmonic dipole, the perturbation Hamiltonian $H^{\prime}$ becomes the dipole-dipole interaction term $H_{A B}=\frac{\mu_{A} \mu_{B}}{r^{3}}\left(3\left(\hat{\mu}_{A} \bullet \hat{r}\right)\left(\hat{\mu}_{B} \bullet \hat{r}\right)-\left(\hat{\mu}_{A} \bullet \hat{\mu}_{B}\right)\right)$, where $\mu_{A}$ and $\mu_{B}$ refer to the plasmonic and electron-hole pair dipoles, respectively. Then, one can obtain the transition probability using Fermi's golden rule:

$$
w_{A B}=\frac{2 \pi}{\hbar^{2}}\left|H_{A B}\right|^{2}\left(\delta\left(E_{A}-E_{B}-\hbar \omega\right)+\delta\left(E_{A}-E_{B}+\hbar \omega\right)\right)
$$

Based on some approximations and simplification, Cushing et al. showed that the full enhancement in the light absorption of a semiconductor can be approximately 10000 for their Au@ $\mathrm{Cu}_{2} \mathrm{O}$ samples. This large increase in light absorption can significantly enhance the solar utilization efficiency. Since this mechanism was proposed, it has been widely used to explain experimentally obtained results $29,40,47,49,107,108$.

As can be seen, the two viewpoints are identical and are based on the same physical principle that the plasmonic dipole is coupled with the electron-hole pair dipole in the semiconductor through the near field it induced. In the first viewpoint, the near field induced by the plasmonic dipole is often explicitly calculated using the FDTD or DDA method. In contrast, in the second viewpoint, the near field is implicitly expressed by a formula.

Based on the plasmon-induced resonance energy transfer mechanism, there are three important factors that can affect the energy transfer efficiency: the overlap between LSPR absorption and optical transitions in the semiconductor, the intensity of the enhanced near-field and the volume in the nearby semiconductor that the enhanced near-field can cover. Generally, the optical transition of a semiconductor can be tuned by selective doping with intrinsic or extrinsic dopants, which has been widely studied and reviewed over the past decades ${ }^{5-9}$. Here, we focus mainly on tuning the properties related to LSPR. Both experimental and theoretical results have shown that the three factors are generally correlated with each other ${ }^{55,58,60}$. Using Ag@AgCl as a model system, we showed that the LSPR absorption can be easily tuned to cover the entire visible light range by varying the size, shape, and dielectric environment of Ag PNPs (Figure 9). Moreover, both the intensity and distribution range of the enhanced near-field change accordingly ${ }^{58}$. In Figure 10, the characteristics of the near-field around Ag nanorods with different aspect ratios are shown. Overall, both the intensity and distribution range of the near-field increase with the LSPR absorption efficiency. In addition, compared with the transverse mode, the longitudinal plasmon mode of silver nanorods is more effective in extending LSPR absorption and increasing the intensity and distribution range of the near 
a

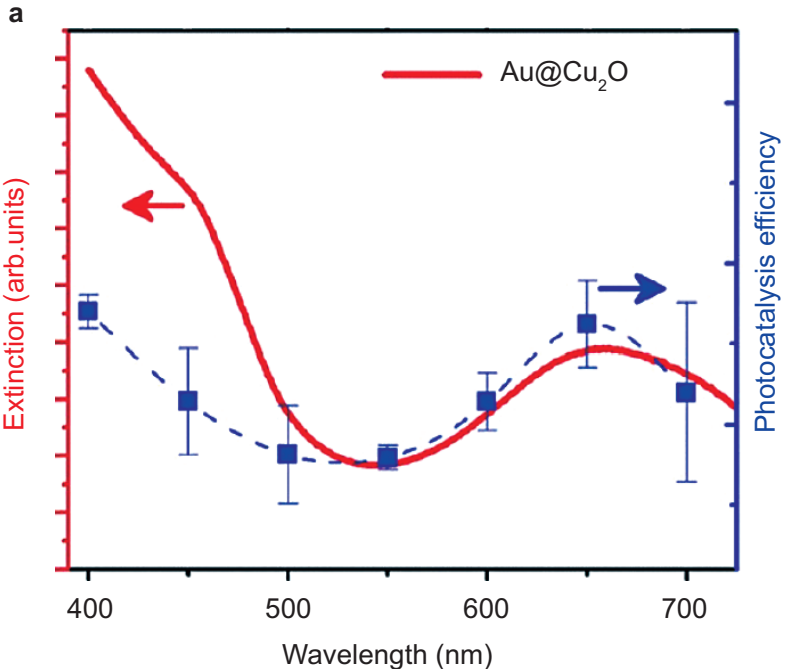

c

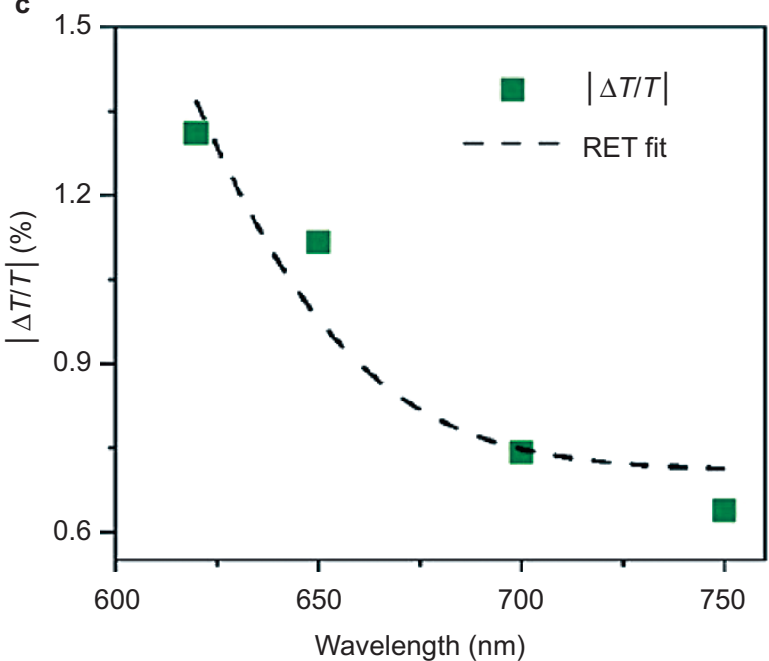

d

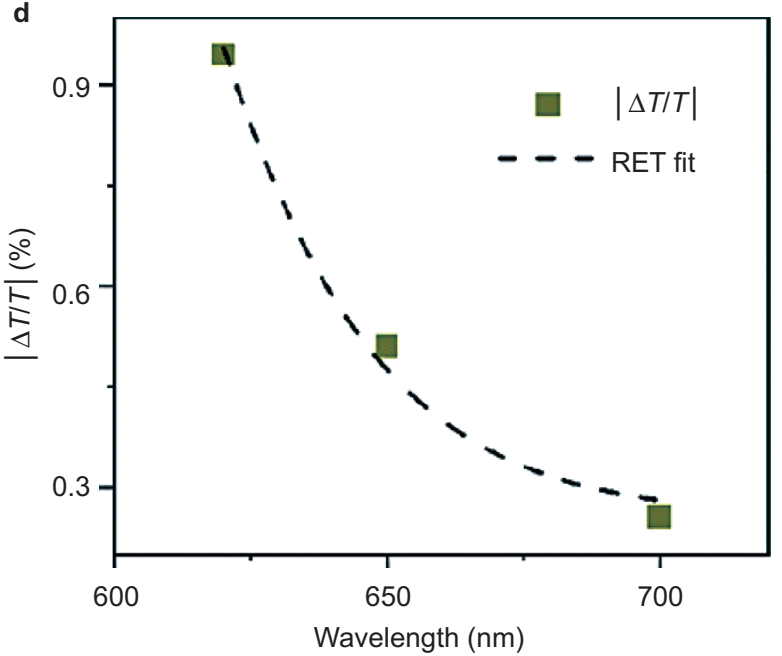

Figure 8 The extinction spectra and photocatalytic action spectra for $\mathrm{Au}_{\mathrm{C}} \mathrm{Cu}_{2} \mathrm{O}(\mathbf{a})$ and $\mathrm{Au}_{\mathrm{SiO}} @ \mathrm{Cu}_{2} \mathrm{O}(\mathbf{b})$. (c) and (d) show the wavelength-dependent signal amplitudes (which measure the carrier density) for $\mathrm{Au} @ \mathrm{Cu}_{2} \mathrm{O}$ and $\mathrm{Au}_{\mathrm{S}} \mathrm{SiO} \mathrm{O}_{2} @ \mathrm{Cu}_{2} \mathrm{O}$, respectively; each result is also fit with the plasmon-induced resonance energy transfer (RET) model on the basis of dipole-dipole interaction rules. Figure reproduced with permission: Ref. 104, ( 2012 ACS.

field. In a recent study, Near et al. showed that there is a modified linear relationship between the wavelength of the longitudinal LSPR mode and the aspect ratio of Au nanorods. Moreover, the relationship between the Au nanorod diameter and extinction intensity is linear within a specific aspect ratio ${ }^{109}$. Similar linear relationships are also found for Ag nanorods ${ }^{110}$. These results can allow for the facile determination of the dimensions of nanorods necessary to generate the desired LSPR characteristics.

Given the benefits of Au nanorods in this energy transfer process, Liu et al. experimentally prepared gold-nanorod-photosensitized $\mathrm{TiO}_{2}{ }^{53}$. They showed that broadband visible light absorption can be easily realized by varying the aspect ratio of Au nanorods. Moreover, the enhancement of activity induced by the longitudinal plasmon mode is indeed larger than that induced by the transverse mode. Li et al. recently reported the preparation of $\mathrm{Au}$ nanorod@ $\mathrm{TiO}_{2}$ yolk-shell nanostructures ${ }^{79}$. Significantly enhanced photocatalytic activity was obtained. Given the ability to convert low energy light at a specific wavelength by tuning the aspect ratio of Au nanorods, they proposed the potential for designing photocatalysts with specific light harvesting. In addition, many other Au/ Ag nanorod-sensitized semiconductor photocatalysts have also been reported with enhanced photocatalytic performance ${ }^{39,87,88,111,112}$.
The benefits of plasmon-induced resonance energy transfer for improving the photocatalytic activities of traditional semiconductor photocatalysts include the following: (i) increasing the rate of electron-hole formation in the regions of semiconductor covered by the near field, and (ii) localizing the generation of electron-hole pairs mostly near the surface of semiconductor; therefore, the recombination during their migration can be significantly decreased. The benefit of (ii) is particularly important for materials that suffer from severe bulk recombination of carriers, such as $\mathrm{Fe}_{2} \mathrm{O}_{3}$. It has been demonstrated that $\mathrm{Au} / \mathrm{Fe}_{2} \mathrm{O}_{3}$ exhibits significantly enhanced solar to fuel energy conversion efficiency ${ }^{46}$. Additionally, in comparison with the hot electron injection energy transfer, this energy transfer is not constrained by the requirements of band alignment, electronic coupling and charge balance, thus offering more flexibility in the selection of materials for photocatalytic applications.

\section{PLASMON-INDUCED RADIATIVE ENERGY TRANSFER}

As introduced above, the LSPR of PNPs can also decay radiatively. The emitted photons may be absorbed by the nearby semiconductor, generating electron-hole pairs, if their energies are large enough. This 

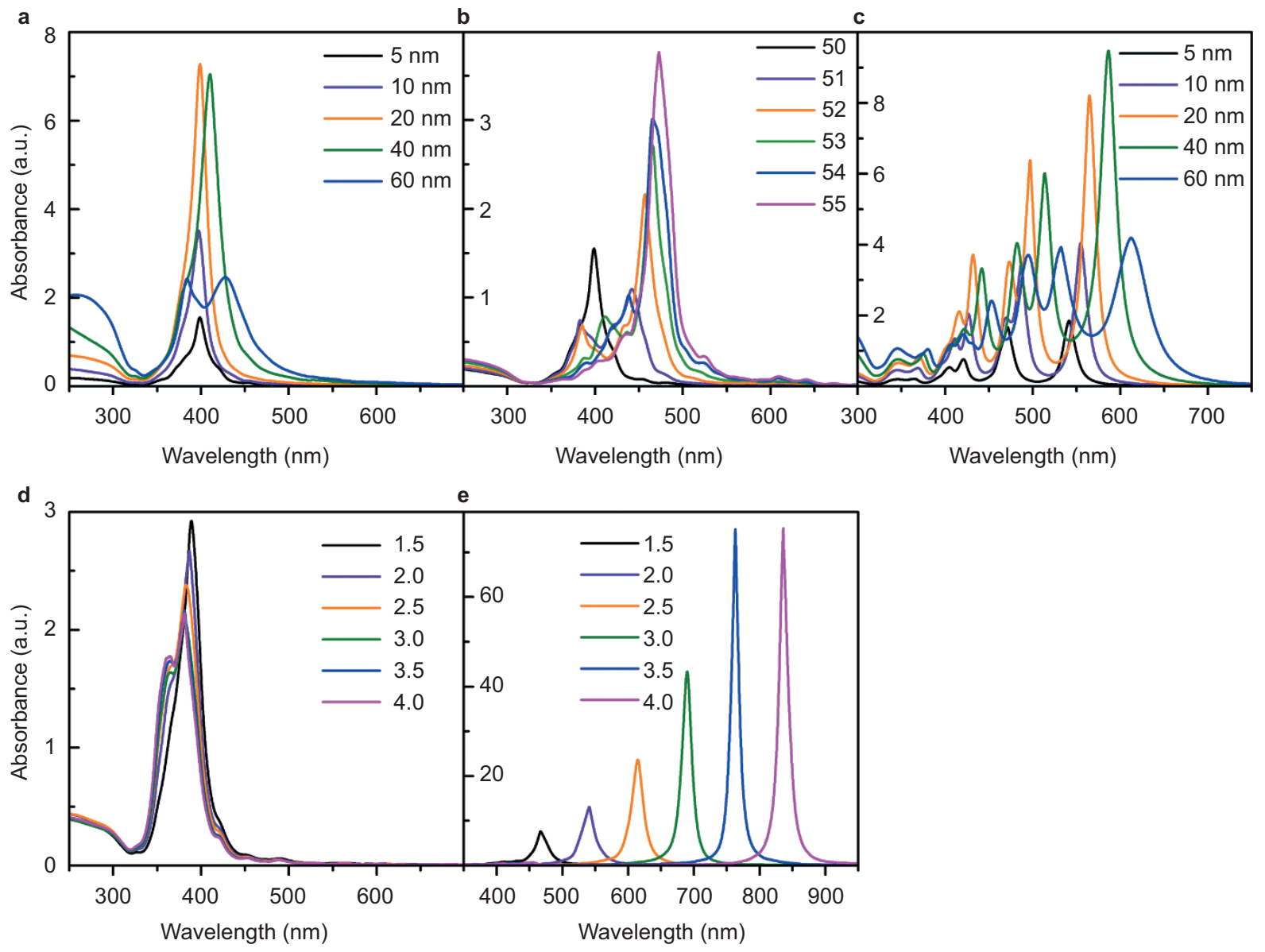

Figure 9 The LSPR absorption efficiencies and wavelengths of different Ag PNPs on AgCl. (a) Ag nanospheres with different diameters. (b) A Ag nanosphere (diameter of $5 \mathrm{~nm}$ ) embedded in $\mathrm{AgCl}$ at different depths. (c) Ag nanocubes with different edge lengths. (d) and (e) are for the transverse and longitudinal modes of silver nanorods with different aspect ratios, respectively. Figure reproduced with permission: Ref. 58, ๔ 2014 ACS.

energy transfer process was indirectly demonstrated by Kumar et al. ${ }^{113}$ They observed that when the $\mathrm{SiO}_{2}$ layer between $\mathrm{Ag}$ and $\mathrm{TiO}_{2}$ is thick enough that the other plasmonic enhancement effects are well ruled out, the photocatalytic activity is still enhanced. The only explanation is thus the plasmon-induced radiative energy transfer. At the early stage of development of plasmonic photocatalysts, this energy transfer mechanism was proposed to explain the experimental results ${ }^{26,42,114}$. However, an increasing number of studies have shown that the contribution of this energy transfer process is very limited. In particular, the radiative efficiency is very low when the PNPs are small ${ }^{57,115,116}$. Dulkeith et al. ${ }^{116}$ showed that the luminescence quantum yield of $\mathrm{Au}$ nanospheres in the range of $2-35 \mathrm{~nm}$ is only on the order of $10^{-6}$. Because plasmonic metal interacts with the semiconductor through the electromagnetic field it induced, Cushing et al. used a full quantum electrodynamics theory to treat this interaction process and concluded that the radiative contribution is virtually negligible ${ }^{104}$.

In contrast, the radiative decay typically dominates in large PNPs. For example, when the size of Ag PNPs exceeds $40 \mathrm{~nm}$, the radiative decay becomes dominant ${ }^{57}$. Nevertheless, it has been shown that the radiative energy transfer contributes only less than $25 \%$ to the enhancement of the photocatalytic performance ${ }^{30,113,117}$. This is probably because the structures of the plasmonic composites are generally irregular, which cannot effectively utilize the scattering effect. In the field of photovoltaics, however, it has been widely demonstrated that the prominent scattering effect of relatively large PNPs can efficiently trap light in the semiconductor layer in elaborately designed solar cells ${ }^{118}$, as schematically shown in Figure 11 . This light trapping can significantly increase the effective optical path length in the semiconductor and thus the light absorption efficiency. Similarly, one may potentially obtain the best radiative energy transfer in plasmonic photocatalysis by optimizing the hybrid structures of plasmonic photocatalysts. This may be more easily realized in the photoelectrode of photoelectrochemical cells because of the relatively easy control of the microstructures.

\section{CONCLUSION AND OUTLOOK}

The unique energy transfer from PNPs to conventional semiconductor photocatalysts is one of the most prominent characteristics of plasmonic photocatalysts. This specialty has established a new paradigm in the harvesting of solar energy for photocatalytic applications. This work reviews and discusses the three energy transfer processes proposed in previous studies. In general, the primary energy transfer processes are plasmon-induced hot electron injection and resonance energy transfer, while plasmon-induced radiative energy transfer is very limited at present. The specific structure of the plasmonic photocatalytic composite can affect both the generation and injection efficiencies of hot electrons, thus determining the energy transfer efficiency based on the plasmon-induced hot electron injection 

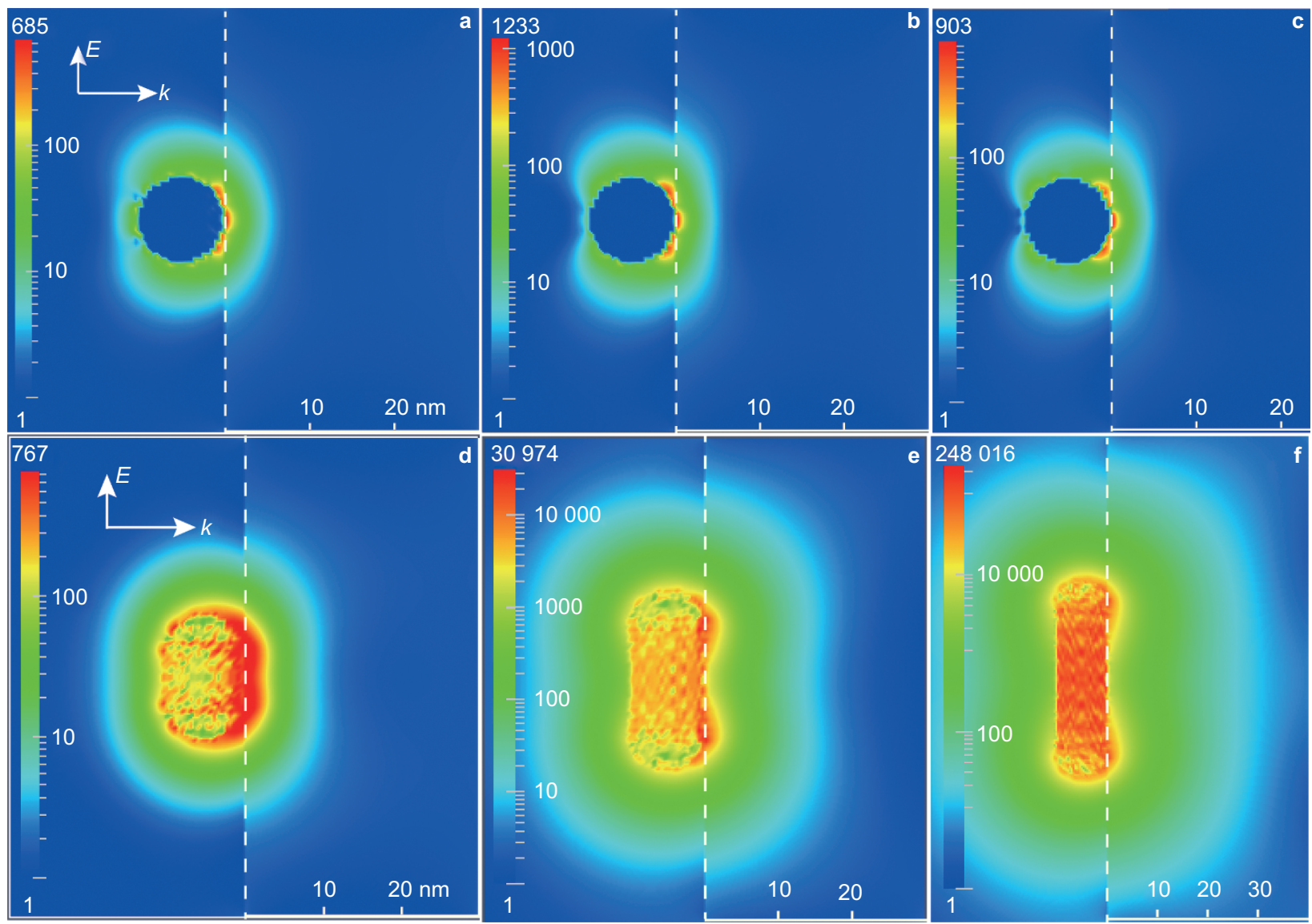

Figure 10 The near-field enhancements (given by $|E|^{2} /\left|E_{0}\right|^{2}$ ) induced by the transverse LSPR-mode of a Ag nanorod with aspect ratios of (a) 1.5, (b) 2.5, and (c) 4.0. (d), (e) and (f) respectively show the corresponding results induced by the longitudinal mode. The depicted cross sections bisect the corresponding Ag nanorods. Figure reproduced with permission: Ref. 58, (c) 2014 ACS.

process. The plasmon-induced resonance energy transfer depends on the near-field enhancement and the overlap between the LSPR absorption and the optical transitions in semiconductors. In addition, this

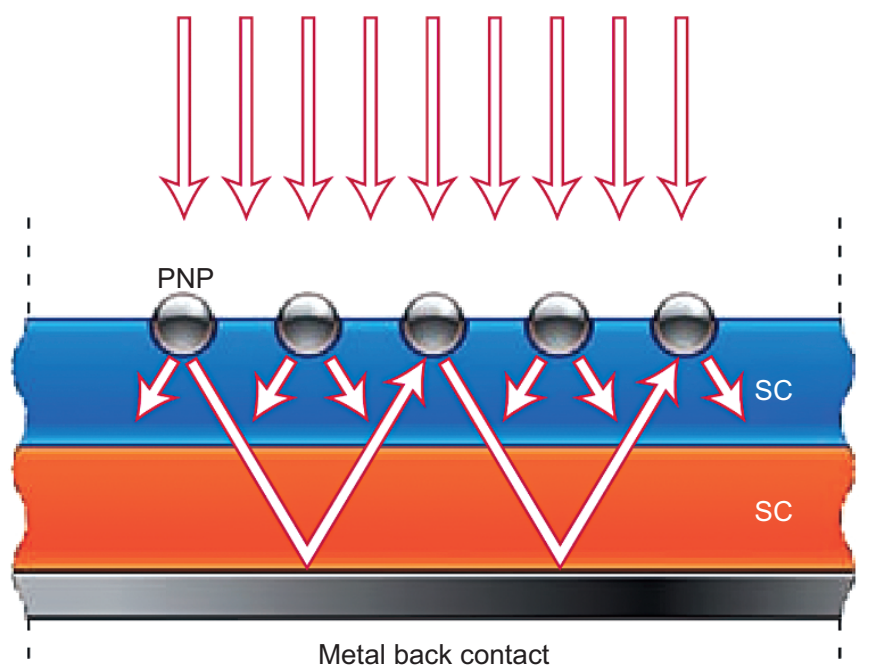

Figure 11 Light trapping in a semiconductor by combining the scattering from PNPs at the surface of a solar cell with reflecting from a metal back contact. Figure adapted with permission: Ref. 118, (c) 2010 NPG. PNP, plasmonic nanoparticle; SC, semiconductor. energy transfer process localizes the generation of electron-hole pairs mainly near the PNPs, which significantly decreases the carrier recombination rate. On the other hand, for the hot electron injection process to occur, many requirements, such as proper band alignment and electronic coupling between the metal and semiconductor and charge balance during photocatalysis, should be satisfied. This imposes strict limitations on the selection of materials for preparing efficient plasmonic photocatalysts. In contrast, the plasmon-induced resonance energy transfer is not constrained by such requirements. With a better understanding of the energy transfer mechanisms, considerably more efficient plasmonic photocatalytic systems may be intentionally devised, which would ultimately enable the industrialization and commercialization of photocatalytic technology.

As a relatively new and burgeoning filed, one can anticipate that new understanding of the physical processes underlying the energy transfer processes will be achieved and that new energy transfer processes may also be proposed. Here, we present an outlook on their development in the near future:

(1) For hot electron injection energy transfer, many factors that affect the generation of hot electrons have been experimentally revealed, including the size, shape, and dielectric environment of PNPs. However, the underlying physics is not well understood. Recently, Govorov et al. developed a series of theories for the dynamic response of typical plasmonic NPs, which can be used to study the generation of hot electrons ${ }^{119-122}$. It is expected that 
the widespread applications of these theories will not only provide a deeper understanding of the factors that affect the generation of hot electrons but also reveal new ones. Additionally, the interface SB is often estimated in the Schottky-Mott limit, which is generally too simple to provide the correct band alignment between the metal and semiconductor. Moreover, the interface chemical and electronic structures often affect the interface SB. Therefore, systemic investigations of the band alignments between plasmonic metals and semiconductors are expected for understanding and optimizing the relevant experimental results. In this respect, first principle studies may contribute substantial information ${ }^{91,93-95}$.

(2) Here, we tentatively propose the potential of direct electron injection from PNPs to semiconductors. However, the detailed interfacial electronic structures require further study to confirm whether this can be an effective energy transfer process in plasmonic photocatalytic composites.

(3) For plasmon-induced resonance energy transfer, there are still different viewpoints. For example, Zhang et al. named this plasmon-induced resonance energy transfer the LSPR-powered bandgap breaking effect ${ }^{30}$. In their picture, the electron collisions at the metal-semiconductor interface excite electrons from the valence band of the semiconductor to the conduction band, thus generating electron-hole pairs. In our opinion, this way of generating electron-hole pairs may indeed occur at the interface, which arises from the non-radiative decay of LSPR and does not contradict the plasmon-induced resonance energy transfer. Instead, it may be regarded as a new energy transfer process. Furthermore, this energy transfer may occur only if the plasmonic metal and semiconductor are in direct contact. In many reports in the literature, the plasmon-induced resonance energy transfer is often also regarded as the near-field enhancement mechanism. This generally confuses new researchers in this field. When reviewing the literature, it is important to note that they are actually identical.

\section{COMPETING INTERESTS}

The authors declare no competing financial interests.

\section{ACKNOWLEDGEMENTS}

This work is supported by the National Basic Research Program of China (973 program, 2013CB632401) and by the National Science Foundation of China (Grant NOs 11374190 and 21333006), the Taishan Scholar Program of Shandong and 111 Project B13029. We also thank the National Supercomputer Center in Jinan for providing high performance computation.

1 Fujishima A, Honda K. Electrochemical photolysis of water at a semiconductor electrode. Nature 1972; 238: 37-38.

2 Chen XB, Shen SH, Guo LJ, Mao SS. Semiconductor-based photocatalytic hydrogen generation. Chem Rev 2010; 110: 6503-6570.

3 Ismail AA, Bahnemann DW. Photochemical splitting of water for hydrogen production by photocatalysis: a review. Sol Energy Mater Sol Cells 2014; 128: 85-101.

4 Nakata K, Fujishima A. $\mathrm{TiO}_{2}$ photocatalysis: design and applications. J Photochem Photobiol C 2012; 13: 169-189.

5 Asahi R, Morikawa T, Ohwaki T, Aoki K, Taga Y. Visible-light photocatalysis in nitrogen-doped titanium oxides. Science 2001; 293: 269-271.

6 Dozzi MV, Selli E. Doping $\mathrm{TiO}_{2}$ with p-block elements: effects on photocatalytic activity. J Photochem Photobiol C 2013; 14: 13-28.

7 Hoffmann MR, Martin ST, Choi W, Bahnemann DW. Environmental applications of semiconductor photocatalysis. Chem Rev 1995; 95: 69-96.

8 Wang SB, Pan L, Song JJ, Mi WB, Zou JJ et al. Titanium-defected undoped anatase $\mathrm{TiO}_{2}$ with $p$-type conductivity, room-temperature ferromagnetism, and remarkable photocatalytic performance. J Am Chem Soc 2015; 137: 2975-2983.
9 Yang KS, Dai Y, Huang BB, Whangbo MH. Density functional characterization of the band edges, the band gap states, and the preferred doping sites of halogen-doped $\mathrm{TiO}_{2}$. Chem Mater 2008; 20: 6528-6534.

$10 \mathrm{Ma} \mathrm{XC}$, Dai Y, Huang BB. Origin of the increased photocatalytic performance of $\mathrm{TiO}_{2}$ nanocrystal composed of pure core and heavily nitrogen-doped shell: a theoretical study. ACS Appl Mater Interfaces 2014; 6: 22815-22822.

11 Odobel F, Pellegrin Y. Recent advances in the sensitization of wide-band-gap nanostructured $p$-type semiconductors. Photovoltaic and photocatalytic applications. J Phys Chem Lett 2013; 4: 2551-2564.

12 Pelaez M, Nolan NT, Pillai SC, Seery MK, Falaras P et al. A review on the visible light active titanium dioxide photocatalysts for environmental applications. App/ Catal B 2012; 125: 331-349.

13 Serpone N, Emeline AV. Semiconductor photocatalysis - past, present, and future outlook. J Phys Chem Lett 2012; 3: 673-677.

14 Ma XC, Dai Y, Guo M, Huang BB. Insights into the role of surface distortion in promoting the separation and transfer of photogenerated carriers in anatase $\mathrm{TiO}_{2}$. J Phys Chem C 2013; 117: 24496-24502.

15 Chen XB, Liu L, Yu PY, Mao SS. Increasing solar absorption for photocatalysis with black hydrogenated titanium dioxide nanocrystals. Science 2011; 331: 746-750.

16 Liu L, Yu PY, Chen XB, Mao SS, Shen DZ. Hydrogenation and disorder in engineered black $\mathrm{TiO}_{2}$. Phys Rev Lett 2013; 111: 065505.

17 Lu JB, Dai Y, Jin H, Huang BB. Effective increasing of optical absorption and energy conversion efficiency of anatase $\mathrm{TiO}_{2}$ nanocrystals by hydrogenation. Phys Chem Chem Phys 2011; 13: 18063-18068.

18 Awazu K, Fujimaki M, Rockstuhl C, Tominaga J, Murakami $\mathrm{H}$ et al. A plasmonic photocatalyst consisting of silver nanoparticles embedded in titanium dioxide. J Am Chem Soc 2008; 130: 1676-1680.

19 Wang P, Huang BB, Qin XY, Zhang XY, Dai Y et al. Ag@AgCl: a highly efficient and stable photocatalyst active under visible light. Angew Chem Int Ed 2008; 47: 79317933.

$20 \mathrm{Li} \mathrm{XN}$, Ju Z, Li F, Huang Y, Xie YM et al. Visible light responsive $\mathrm{Bi}_{7} \mathrm{Fe}_{3} \mathrm{Ti}_{3} \mathrm{O}_{21}$ nanoshelf photocatalysts with ferroelectricity and ferromagnetism. J Mater Chem $\mathrm{A}$ 2014; 2: 13366-13372.

21 Wang WJ, Huang BB, Ma XC, Wang ZY, Qin XY et al. Efficient separation of photogenerated electron-hole pairs by the combination of a heterolayered structure and internal polar field in pyroelectric $\mathrm{BiOlO}_{3}$ nanoplates. Chem Eur J 2013; 19 : 14777-14780.

22 Zhang R, Dai Y, Lou ZZ, Li ZJ, Wang ZY et al. Layered photocatalyst $\mathrm{Bi}_{2} \mathrm{O}_{2}\left[\mathrm{BO}_{2}(\mathrm{OH})\right]$ nanosheets with internal polar field enhanced photocatalytic activity. Cryst Eng Comm 2014; 16: 4931-4934.

23 Batzill M. Fundamental aspects of surface engineering of transition metal oxide photocatalysts. Energy Environ Sci 2011; 4: 3275-3286.

24 Xiang QJ, Yu JG, Jaroniec M. Graphene-based semiconductor photocatalysts. Chem Soc Rev 2012; 41: 782-796.

25 Xiang QJ, Yu JG. Graphene-based photocatalysts for hydrogen generation. J Phys Chem Lett 2013; 4: 753-759.

26 Linic S, Christopher P, Ingram DB. Plasmonic-metal nanostructures for efficient conversion of solar to chemical energy. Nat Mater 2011; 10: 911-921.

27 Clavero C. Plasmon-induced hot-electron generation at nanoparticle/metal-oxide interfaces for photovoltaic and photocatalytic devices. Nat Photon 2014; 8: 95-103.

28 Cheng HF, Fuku K, Kuwahara Y, Mori K, Yamashita H. Harnessing single-active plasmonic nanostructures for enhanced photocatalysis under visible light. J Mater Chem A 2015; 3: 5244-5258.

29 Lou ZZ, Wang ZY, Huang BB, Dai Y. Synthesis and activity of plasmonic photocatalysts. Chem Cat Chem 2014; 6: 2456-2476.

30 Zhang XM, Chen YL, Liu RS, Tsai DP. Plasmonic photocatalysis. Rep Prog Phys 2013; 76: 046401.

31 Wang P, Huang BB, Dai Y, Whangbo MH. Plasmonic photocatalysts: harvesting visible light with noble metal nanoparticles. Phys Chem Chem Phys 2012; 14: 9813-9825.

32 Hou WB, Cronin SB. A review of surface plasmon resonance-enhanced photocatalysis. Adv Funct Mater 2013; 23: 1612-1619.

33 Jiang RB, Li BX, Fang CH, Wang JF. Metal/semiconductor hybrid nanostructures for plasmon-enhanced applications. Adv Mater 2014; 26: 5274-5309.

34 Brongersma ML, Halas NJ, Nordlander P. Plasmon-induced hot carrier science and technology. Nat Nano 2015; 10: 25-34.

35 Ma XC, Dai Y, Yu L, Huang BB. Noble-metal-free plasmonic photocatalyst: hydrogen doped semiconductors. Sci Rep 2014; 4: 3986.

36 Wang Z, Yang CY, Lin TQ, Yin H, Chen P et al. H-doped black titania with very high solar absorption and excellent photocatalysis enhanced by localized surface plasmon resonance. Adv Funct Mater 2013; 23: 5444-5450.

37 Zheng ZK, Huang BB, Qin XY, Zhang XY, Dai Y et al. Facile in situ synthesis of visiblelight plasmonic photocatalysts $\mathrm{M@TiO}_{2}(\mathrm{M}=\mathrm{Au}, \mathrm{Pt}, \mathrm{Ag})$ and evaluation of their photocatalytic oxidation of benzene to phenol. J Mater Chem 2011; 21: 9079-9087.

38 Yang D, Sun YY, Tong ZW, Tian Y, Li YB et al. Synthesis of $\mathrm{Ag} / \mathrm{TiO}_{2}$ nanotube heterojunction with improved visible-light photocatalytic performance inspired by bioadhesion. J Phys Chem C 2015; 119: 5827-5835.

39 Wang XT, Liow C, Qi DP, Zhu BW, Leow WR et al. Programmable photoelectrochemical hydrogen evolution based on multi-segmented CdS-Au nanorod arrays. Adv Mater 2014; 26: 3506-3512.

40 Solarska R, Bienkowski K, Zoladek S, Majcher A, Stefaniuk T et al. Enhanced water splitting at thin film tungsten trioxide photoanodes bearing plasmonic goldpolyoxometalate particles. Angew Chem 2014; 53: 14196-14200. 
41 Sellappan R, Nielsen MG, González-Posada F, Vesborg PCK, Chorkendorff I et al. Effects of plasmon excitation on photocatalytic activity of $\mathrm{Ag} / \mathrm{TiO}_{2}$ and $\mathrm{Au} / \mathrm{TiO}_{2}$ nanocomposites. J Catal 2013; 307: 214-221.

42 Christopher P, Ingram DB, Linic S. Enhancing photochemical activity of semiconductor nanoparticles with optically active Ag nanostructures: photochemistry mediated by $\mathrm{Ag}$ surface plasmons. J Phys Chem C 2010; 114: 9173-9177.

43 Zhang L, Blom DA, Wang $\mathrm{H}$. Au- $\mathrm{Cu}_{2} \mathrm{O}$ core-shell nanoparticles: a hybrid metalsemiconductor heteronanostructure with geometrically tunable optical properties. Chem Mater 2011; 23: 4587-4598.

44 Yu KF, Tian Y, Tatsuma T. Size effects of gold nanaoparticles on plasmon-induced photocurrents of gold- $\mathrm{TiO}_{2}$ nanocomposites. Phys Chem Chem Phys 2006; 8: 54175420.

45 Torimoto T, Horibe H, Kameyama T, Okazaki K, Ikeda S et al. Plasmon-enhanced photocatalytic activity of cadmium sulfide nanoparticle immobilized on silicacoated gold particles. J Phys Chem Lett 2011; 2: 2057-2062.

46 Thomann I, Pinaud BA, Chen ZB, Clemens BM, Jaramillo TF et al. Plasmon enhanced solar-to-fuel energy conversion. Nano Lett 2011; 11: 3440-3446.

47 Shi XW, Ji YL, Hou S, Liu WQ, Zhang $\mathrm{H}$ et al. Plasmon enhancement effect in Au gold nanorods@Cu20 core-shell nanostructures and their use in probing defect states. Langmuir 2015; 31: 1537-1546.

48 Seh ZW, Liu SH, Low M, Zhang SY, Liu ZL et al. Janus Au-TiO 2 photocatalysts with strong localization of plasmonic near-fields for efficient visible-light hydrogen generation. Adv Mater 2012; 24: 2310-2314.

49 Ren ST, Wang BY, Zhang H, Ding P, Wang Q. Sandwiched ZnO@Au@Cu $\mathrm{Cu}_{2}$ nanorod films as efficient visible-light-driven plasmonic photocatalysts. ACS App/ Mater Interfaces 2015; 7: 4066-4074.

50 Ma X, Zhao K, Tang HJ, Chen Y, Lu CG et al. New insight into the role of gold nanoparticles in Au@CdS core-shell nanostructures for hydrogen evolution. Small 2014; 10: 4664-4670.

51 Mubeen S, Lee J, Singh N, Krämer S, Stucky GD et al. An autonomous photosynthetic device in which all charge carriers derive from surface plasmons. Nat Nano 2013; 8: 247-251.

52 Long R, Prezhdo OV. Instantaneous generation of charge-separated state on $\mathrm{TiO}_{2}$ surface sensitized with plasmonic nanoparticles. J Am Chem Soc 2014; 136: 4343-4354.

53 Liu LQ, Ouyang SX, Ye JH. Gold-nanorod-photosensitized titanium dioxide with widerange visible-light harvesting based on localized surface plasmon resonance. Angew Chem Int Ed 2013; 52: 6689-6693.

54 Fan XF, Zheng WT, Singh DJ. Light scattering and surface plasmons on small spherical particles. Light Sci Appl 2014; 3: e179.

55 Kelly KL, Coronado E, Zhao LL, Schatz GC. The optical properties of metal nanoparticles: the influence of size, shape, and dielectric environment. J Phys Chem B 2002; 107: 668-677.

56 Wang $\mathrm{CL}$, Astruc D. Nanogold plasmonic photocatalysis for organic synthesis and clean energy conversion. Chem Soc Rev 2014; 43: 7188-7216.

57 Evanoff DD, Chumanov G. Synthesis and optical properties of silver nanoparticles and arrays. Chem Phys Chem 2005; 6: 1221-1231.

58 MaXC, Dai Y, Yu L, Lou ZZ, Huang BB et al. Electron-hole pair generation of the visiblelight plasmonic photocatalyst $\mathrm{Ag} @ \mathrm{AgCl}$ : enhanced optical transitions involving midgap defect states of AgCl. J Phys Chem C 2014; 118: 12133-12140.

59 Burda C, Chen XB, Narayanan R, El-Sayed MA. Chemistry and properties of nanocrystals of different shapes. Chem Rev 2005; 105: 1025-1102.

60 Mahmoud MA, Chamanzar M, Adibi A, El-Sayed MA. Effect of the dielectric constant of the surrounding medium and the substrate on the surface plasmon resonance spectrum and sensitivity factors of highly symmetric systems: silver nanocubes. J Am Chem Soc 2012; 134: 6434-6442.

61 Hovel H, Fritz S, Hilger A, Kreibig U, Vollmer M. Width of cluster plasmon resonances: bulk dielectric functions and chemical interface damping. Phys Rev B 1993; 48: 18178-18188.

62 Endriz JG, Spicer WE. Surface-plasmon-one-electron decay and its observation in photoemission. Phys Rev Lett 1970; 24: 64-68.

63 Lehmann J, Merschdorf M, Pfeiffer W, Thon A, Voll S et al. Surface plasmon dynamics in silver nanoparticles studied by femtosecond time-resolved photoemission. Phys Rev Lett 2000; 85: 2921-2924.

64 Hodak JH, Martini I, Hartland GV. Spectroscopy and dynamics of nanometer-sized noble metal particles. J Phys Chem B 1998; 102: 6958-6967.

65 Liu ZW, Hou WB, Pavaskar P, Aykol M, Cronin SB. Plasmon resonant enhancement of photocatalytic water splitting under visible illumination. Nano Lett2011; 11: 11111116.

66 Manjavacas A, Liu JG, Kulkarni V, Nordlander P. Plasmon-induced hot carriers in metallic nanoparticles. ACS Nano 2014; 8: 7630-7638.

67 Mubeen S, Hernandez-Sosa G, Moses D, Lee J, Moskovits M. Plasmonic photosensitization of a wide band gap semiconductor: converting plasmons to charge carriers. Nano Lett 2011; 11: 5548-5552.

68 Grätzel M. Photoelectrochemical cells. Nature 2001; 414: 338-344.

69 Hashimoto $\mathrm{K}$, Irie $\mathrm{H}$, Fujishima $\mathrm{A} . \mathrm{TiO}_{2}$ photocatalysis: a historical overview and future prospects. Jpn J Appl Phys 2005; 44: 8269.

70 Sundararaman R, Narang P, Jermyn AS, Goddard III WA, Atwater HA. Theoretical predictions for hot-carrier generation from surface plasmon decay. Nat Commun 2014; 5: 5788.

71 Bernardi M, Mustafa J, Neaton JB, Louie SG. Theory and computation of hot carriers generated by surface plasmon polaritons in noble metals. Nat Commun 2015; 6: 7044.
72 Zhao GL, Kozuka H, Yoko T. Sol-gel preparation and photoelectrochemical properties of $\mathrm{TiO}_{2}$ films containing Au and Ag metal particles. Thin Solid Films 1996; 277: 147-154.

73 Ohko Y, Tatsuma T, Fujii T, Naoi K, Niwa C et al. Multicolour photochromism of $\mathrm{TiO}_{2}$ films loaded with silver nanoparticles. Nat Mater 2003; 2: 29-31.

74 Furube A, Du LC, Hara K, Katoh R, Tachiya M. Ultrafast plasmon-induced electron transfer from gold nanodots into $\mathrm{TiO}_{2}$ nanoparticles. J Am Chem Soc 2007; 129 : $14852-14853$

75 Asbury JB, Hao EC, Wang YQ, Ghosh HN, Lian TQ. Ultrafast electron transfer dynamics from molecular adsorbates to semiconductor nanocrystalline thin films. J Phys Chem B 2001; 105: 4545-4557.

76 DuChene JS, Sweeny BC, Johnston-Peck AC, Su D, Stach EA et al. Prolonged hot electron dynamics in plasmonic-metal/semiconductor heterostructures with implications for solar photocatalysis. Angew Chem Int Ed 2014; 53: 7887-7891.

77 Liu LQ, Li P, Wang T, Hu HL, Jiang HY et al. Constructing a multicomponent junction for improved visible-light photocatalytic performance induced by Au nanoparticles. Chem Commun 2015; 51: 2173-2176.

78 Ha JW, Ruberu TPA, Han R, Dong B, Vela J et al. Super-resolution mapping of photogenerated electron and hole separation in single metal-semiconductor nanocatalysts. J Am Chem Soc 2014; 136: 1398-1408.

79 Li A, Zhang P, Chang XX, Cai WT, Wang T et al. Gold nanorod@TiO 2 yolk-shell nanostructures for visible-light-driven photocatalytic oxidation of benzyl alcohol. Small 2015; 11: 1892-1899.

80 Tung RT. The physics and chemistry of the Schottky barrier height. App/ Phys Rev 2014; 1: 011304.

81 Park JY, Kim SM, Lee H, Naik B. Hot electron and surface plasmon-driven catalytic reaction in metal-semiconductor nanostructures. Catal Lett2014; 144: 1996-2004.

82 Watanabe A, Kozuka H. Photoanodic properties of sol-gel-derived $\mathrm{Fe}_{2} \mathrm{O}_{3}$ thin films containing dispersed gold and silver particles. J Phys Chem B 2003; 107: 1271312720 .

83 Giugni A, Torre B, Toma A, Francardi M, Malerba M et al. Hot-electron nanoscopy using adiabatic compression of surface plasmons. Nat Nano 2013; 8: 845-852.

84 Li JT, Cushing SK, Zheng P, Meng FK, Chu D et al. Plasmon-induced photonic and energy-transfer enhancement of solar water splitting by a hematite nanorod array. Nat Commun 2013; 4: 2651.

85 Knight MW, Sobhani H, Nordlander P, Halas NJ. Photodetection with active optical antennas. Science 2011; 332: 702-704

86 Knight MW, Wang Y, Urban AS, Sobhani A, Zheng BY et al. Embedding plasmonic nanostructure diodes enhances hot electron emission. Nano Lett2013; 13: 1687-1692.

87 Fang $\mathrm{CH}$, Jia HL, Chang S, Ruan QF, Wang P et al. (Gold core)/(titania shell) nanostructures for plasmon-enhanced photon harvesting and generation of reactive oxygen species. Energy Environ Sci 2014; 7: 3431-3438.

88 Li BX, Gu T, Ming T, Wang JX, Wang P et al. (Gold core)@(ceria shell) nanostructures for plasmon-enhanced catalytic reactions under visible light. ACS Nano 2014; 8: 8152-8162

89 Liu LQ, Dao TD, Kodiyath R, Kang Q, Abe H et al. Plasmonic Janus-composite photocatalyst comprising $\mathrm{Au}$ and $\mathrm{C}-\mathrm{TiO}_{2}$ for enhanced aerobic oxidation over a broad visible-light range. Adv Funct Mater 2014; 24: 7754-7762.

90 Fan XM, Xu CX, Hao XL, Tian ZS, Lin Y. Synthesis and optical properties of Janus structural ZnO/Au nanocomposites. EPL 2014; 106: 67001.

91 Ma XC, Dai Y, Yu L, Huang BB. New basic insights into the low hot electron injection efficiency of gold-nanoparticle-photosensitized titanium dioxide. ACS Appl Mater Interfaces 2014; 6: 12388-12394.

92 Li JT, Cushing SK, Zheng P, Senty T, Meng FK et al. Solar hydrogen generation by a $\mathrm{CdS}-\mathrm{Au}-\mathrm{TiO}_{2}$ sandwich nanorod array enhanced with Au nanoparticle as electron relay and plasmonic photosensitizer. J Am Chem Soc 2014; 136: 8438-8449.

93 Mrovec M, Albina JM, Meyer B, Elsässer C. Schottky barriers at transition-metal/ $\mathrm{SrTiO}_{3}$ (001) interfaces. Phys Rev B 2009; 79: 245121.

94 Rao FY, Kim M, Freeman AJ, Tang SP, Anthony M. Structural and electronic properties of transition-metal/BaTiO ${ }_{3}$ (001) interfaces. Phys Rev B 1997; 55: 13953-13960.

95 Tamura T, Ishibashi S, Terakura K, Weng HM. First-principles study of the rectifying properties of $\mathrm{Pt} / \mathrm{TiO}_{2}$ interface. Phys Rev B 2009; 80: 195302.

96 Smith JG, Faucheaux JA, Jain PK. Plasmon resonances for solar energy harvesting: a mechanistic outlook. Nano Today 2015; 10: 67-80.

97 Lin ZJ, Wang XH, Liu J, Tian ZY, Dai LC et al. On the role of localized surface plasmon resonance in UV-Vis light irradiated $\mathrm{Au} / \mathrm{TiO}_{2}$ photocatalysis systems: pros and cons. Nanoscale 2015; 7: 4114-4123.

98 Persson BNJ. Polarizability of small spherical metal particles: influence of the matrix environment. Surf Sci 1993; 281: 153-162.

99 Bosbach J, Hendrich C, Stietz F, Vartanyan T, Träger F. Ultrafast dephasing of surface plasmon excitation in silver nanoparticles: influence of particle size, shape, and chemical surrounding. Phys Rev Lett 2002; 89: 257404.

100 Petek H, Weida MJ, Nagano H, Ogawa S. Real-time observation of adsorbate atom motion above a metal surface. Science 2000; 288: 1402-1404.

101 Jain PK, Qian W, El-Sayed MA. Ultrafast cooling of photoexcited electrons in gold nanoparticle-thiolated DNA conjugates involves the dissociation of the gold-thiol bond. J Am Chem Soc 2006; 128: 2426-2433.

102 Kale MJ, Avanesian T, Xin HL, Yan J, Christopher P. Controlling catalytic selectivity on metal nanoparticles by direct photoexcitation of adsorbate-metal bonds. Nano Lett 2014; 14: 5405-5412.

103 Kale MJ, Avanesian T, Christopher P. Direct photocatalysis by plasmonic nanostructures. ACS Catal 2014; 4: 116-128. 
104 Cushing SK, Li JT, Meng FK, Senty TR, Suri S et al. Photocatalytic activity enhanced by plasmonic resonant energy transfer from metal to semiconductor. J Am Chem Soc 2012; 134: 15033-15041.

105 Sahoo H. Forster resonance energy transfer - a spectroscopic nanoruler: principle and applications. J Photochem Photobiol C 2011; 12: 20-30.

106 Dressel M, Grüner G. Electrodynamics of solids: optical properties of electrons in matter. Cambridge: Cambridge University Press; 2002.

107 Liu TX, Li BX, Hao YG, Han F, Zhang LL et al. A general method to diverse silver/ mesoporous-metal-oxide nanocomposites with plasmon-enhanced photocatalytic activity. App/ Catal B 2015; 165: 378-388.

108 Meng FK, Cushing SK, Li JT, Hao SM, Wu NQ. Enhancement of solar hydrogen generation by synergistic interaction of $\mathrm{La}_{2} \mathrm{Ti}_{2} \mathrm{O}_{7}$ photocatalyst with plasmonic gold nanoparticles and reduced graphene oxide nanosheets. ACS Catal 2015; 5: 1949-1955.

109 Near RD, Hayden SC, El-Sayed MA. Thin to thick, short to long: spectral properties of gold nanorods by theoretical modeling. J Phys Chem C 2013; 117: 18653-18656.

110 Mahmoud MA, El-Sayed MA. Different plasmon sensing behavior of silver and gold nanorods. J Phys Chem Lett 2013; 4: 1541-1545.

111 Chen HJ, Shao L, Li Q, Wang JF. Gold nanorods and their plasmonic properties. Chem Soc Rev 2013; 42: 2679-2724.

112 Seh ZW, Liu SH, Zhang SY, Bharathi MS, Ramanarayan H et al. Anisotropic growth of titania onto various gold nanostructures: synthesis, theoretical understanding, and optimization for catalysis. Angew Chem Int Ed 2011; 50: 10140-10143.

113 Kumar MK, Krishnamoorthy S, Tan LK, Chiam SY, Tripathy S et al. Field effects in plasmonic photocatalyst by precise $\mathrm{SiO}_{2}$ thickness control using atomic layer deposition. ACS Catal 2011; 1: 300-308.

114 Ingram DB, Christopher P, Bauer JL, Linic S. Predictive model for the design of plasmonic metal/semiconductor composite photocatalysts. ACS Catal 2011; 1: 1441-1447.

115 Mohamed MB, Volkov V, Link S, El-Sayed MA. The 'lightning' gold nanorods: fluorescence enhancement of over a million compared to the gold metal. Chem Phys Lett 2000; 317: 517-523.
116 Dulkeith E, Niedereichholz T, Klar TA, Feldmann J, von Plessen G et al. Plasmon emission in photoexcited gold nanoparticles. Phys Rev B 2004; 70: 205424.

117 Ingram DB, Linic S. Water splitting on composite plasmonic-metal/semiconductor photoelectrodes: evidence for selective plasmon-induced formation of charge carriers near the semiconductor surface. J Am Chem Soc 2011; 133: 5202-5205.

118 Atwater HA, Polman A. Plasmonics for improved photovoltaic devices. Nat Mater 2010; 9: 205-213.

119 Govorov AO, Zhang H. Kinetic density functional theory for plasmonic nanostructures: breaking of the plasmon peak in the quantum regime and generation of hot electrons. J Phys Chem C 2015; 119: 6181-6194.

120 Govorov AO, Zhang H, Demir HV, Gun'ko YK. Photogeneration of hot plasmonic electrons with metal nanocrystals: quantum description and potential applications. Nano Today 2014; 9: 85-101.

121 Govorov AO, Zhang H, Gun'ko YK. Theory of photoinjection of hot plasmonic carriers from metal nanostructures into semiconductors and surface molecules. J Phys Chem C 2013; 117: 16616-16631.

122 Zhang H, Govorov AO. Optical generation of hot plasmonic carriers in metal nanocrystals: the effects of shape and field enhancement. J Phys Chem C 2014; 118: 7606-7614.

\section{(c) (i) (5) $\Theta$ This work is licensed under a Creative Commons Attribution-}

cc. ${ }_{\mathrm{BY}} \mathrm{NC}$ ND NonCommercial-NoDerivs 4.0 Unported License. The images or other third party material in this article are included in the article's Creative Commons license, unless indicated otherwise in the credit line; if the material is not included under the Creative Commons license, users will need to obtain permission from the license holder to reproduce the material. To view a copy of this license, visit http://creativecommons.org/licenses/ by-nc-nd/4.0/ 